PALEO

Revue d'archéologie préhistorique

$27 \mid 2016$

Varia

\title{
Processus géologiques de formation du site d'Isturitz (Sud-Ouest de la France)
}

Implications archéologiques

Geological formation processes of the site of Isturitz (South-West France).

Archaeological implications

Arnaud Lenoble et Jean-Pierre Texier

\section{OpenEdition}

Journals

Édition électronique

URL : http://journals.openedition.org/paleo/3138

DOI : $10.4000 /$ paleo.3138

ISSN : 2101-0420

Éditeur

SAMRA

Édition imprimée

Date de publication : 30 décembre 2016

Pagination : 235-252

ISSN : 1145-3370

Référence électronique

Arnaud Lenoble et Jean-Pierre Texier, « Processus géologiques de formation du site d'Isturitz (Sud-

Ouest de la France) », PALEO [En ligne], 27 | 2016, mis en ligne le 01 juin 2018, consulté le 07 juillet 2020. URL : http://journals.openedition.org/paleo/3138; DOI : https://doi.org/10.4000/paleo.3138

\section{(c) (i) (9)}

PALEO est mis à disposition selon les termes de la licence Creative Commons Attribution - Pas d'Utilisation Commerciale - Pas de Modification 4.0 International. 


\title{
Processus géologiques de formation du site d'Isturitz (Sud-Ouest de la France). Implications archéologiques ${ }^{1}$
}

\author{
Arnaud LENOBLE ${ }^{(a)}$, Jean-Pierre TEXIER ${ }^{(a)}$
}

Résumé : Cet article fait le bilan des travaux géoarchéologiques menés dans le site d'lsturitz au cours des années 19961997 et 2000-2005. L'étude du contexte géomorphologique et des différents affleurements localisés autour et à l'intérieur du massif karstique a permis de retracer les principales phases évolutives du réseau. Celui-ci comprend trois niveaux étagés qui sont reliés à des étapes de creusement de la vallée de l'Arberoue. Une attention particulière a été portée au niveau supérieur dans lequel ont été menées les fouilles. Dans cette partie du réseau, la sédimentation évolue globalement d'un pôle dominé par des écoulements de haute énergie (rivière à écoulement torrentiel, puis ruisseau souterrain), vers un pôle dominé par le ruissellement puis par l'éboulisation. Dans la partie sommitale de l'accumulation, au sein des éboulis, a été détecté un hiatus sédimentaire, contemporain d'une phase très froide du SIO 2. Au cours de cet épisode, s'est développé un cryosol qui s'est notamment traduit par d'importantes déformations des niveaux antérieurs. La séquence se termine par la mise en place d'un plancher stalagmitique au cours de l'Holocène.

Les niveaux proto-aurignaciens et aurignaciens qui ont fait l'objet des fouilles sont associés aux dépôts de ruissellement et aux éboulis de la partie supérieure du remplissage. Une étude taphonomique spécifique a porté sur ces nappes de vestiges. Elle a montré 1) que le gel n'a eu qu'une incidence mineure sur la préservation des niveaux en comparaison du rôle joué par le ruissellement, 2) que le matériel archéologique recueilli dans l'unité II résulte de phénomènes de remaniement et 3) que les niveaux identifiés dans l'unité III correspondent alternativement soit à des lots de pièces déplacées, soit à des ensembles archéologiques supportant l'hypothèse d'une bonne préservation. Les implications de cette étude sont discutées et l'on souligne la nécessité de rechercher les remontages d'intérêt stratigraphique pour valider l'interprétation archéostratigraphique de la séquence.

Mots-clés : Sud-Ouest de la France, karst, processus de formation des sites, taphonomie, ruissellement, cryosol, ProtoAurignacien, Aurignacien.

Abstract: Geological formation processes of the site of Isturitz (South-West France). Archaeological implications. This paper reports on geoarchaeological work conducted at the site of Isturitz during the 1996-1997 and the 2000-2005 excavations. The study of geomorphological context and sedimentary deposits both outside and inside the karstic block permit the main evolutionary phases to be described.

The karstic system comprises three stepped levels, which are linked to downcutting stages of the Arberoue valley. Special attention has been paid to the upper level of the karstic system, i.e. the Saint-Martin-Isturitz level, in which excavations were conducted. Here, sedimentation was initially associated with high-energy flows (torrentially-flowing river followed by an intermittently active underground stream), and later by runoff and rockfall processes. In the upper part of

(a) PACEA, UMR CNRS 5199, Université de Bordeaux, Bât. B18 - Allée Geoffroy Saint-Hilaire CS 50023 - FR- 33615 Pessac cedex arnaud.lenoble@u-bordeaux.fr ; j-pierre.texier@wanadoo.fr

(1) Cet article a été rédigé en 2005 à la suite de la table ronde qui s'est tenue à Hasparren en 2003. Celle-ci avait pour objet d'établir le bilan des recherches effectuées sur la séquence paléolithique de la grotte d'Isturitz. Nous présentons ici les résultats géomorphologiques, lithostratigraphiques et géoarchéologiques acquis à cette date. 
the karstic filling, a sedimentary hiatus, contemporaneous with a very cold phase of OIS 2 , has been identified in the rockfall deposits. This climatic episode is characterized by a cryosol that has notably deformed former sedimentary levels. The sequence ends with a flowstone that accumulated during the Holocene.

The Proto-Aurignacian and Aurignacian levels are buried in runoff and rockfall deposits of the upper part of the fill. They were the focus of a detailed taphonomic study, which demonstrated that 1) freezing has had only minor effects on preservation of these levels compared to the role played by runoff, 2) the presence of artefacts in unit II is the result of reworking processes and, 3) archaeological levels identified in unit III represent either sets of reworked pieces or intact artefact assemblages admitting the assumption of a good preservation. Implications of this study are discussed and it is suggested that refitting analyses should be performed to confirm the site's proposed archaeostratigraphic sequence.

Key-words: South-West France, karst, site formation processes, taphonomy, runoff, cryosol, Proto-Aurignacian, Aurignacian.

\section{Introduction}

Les travaux archéologiques entrepris depuis 1996 dans le site d'Isturitz, d'abord sous la responsabilité d'A. Turq (1996-1997) puis sous celle de Ch. Normand (2000-2005), nous ont donné l'occasion de mener une étude géologique approfondie de la cavité, de son remplissage et de son environnement géomorphologique.

Les principaux objectifs visés étaient les suivants : comprendre l'évolution générale du système karstique, établir la lithostratigraphie des dépôts du remplissage, en caractériser la genèse et évaluer l'impact des processus géologiques sur l'intégrité des assemblages archéologiques contenus dans les sédiments. Nous présentons ci-après un bilan résumé des principaux résultats obtenus dans ces différents domaines.

\section{1 - Méthodes d'étude}

L'étude du contexte géomorphologique du site a été abordée par des travaux de terrain ainsi que par l'analyse des cartes topographique à $1 / 25000$ et géologique à $1 / 50000$ d'Iholdy.

Les coupes, replacées dans leur contexte morphologique, ont été décrites en termes de faciès. Ces derniers ont été définis principalement à partir de critères directement appréciables sur le terrain : organisation générale des dépôts (dépôts massifs, à stratification régulière ou irrégulière), caractéristiques de la stratification (épaisseur, pente, continuité, morphologie et type de contact des différentes strates), structure des sédiments (ouverte, colmatée à support clastique, à support matriciel), présence de granoclassements, granulométrie, morphologie et fabrique des cailloux.

Les analyses granulométriques ont porté sur la matrice des dépôts (i.e. les particules inférieures à $2 \mathrm{~mm}$ ) ainsi que sur les objets archéologiques. Sur les sédiments matriciels, elles ont été réalisées par diffractométrie laser (appareil de type Malvern 2600) et par tamisage mécanique. Les objets archéologiques côtés et recueillis au tamisage ont été mesurés au pied à coulisse ou passés au tamis de façon à établir la proportion d'objets pour trois classes dimensionnelles particulières : objets arrêtés par les tamis de maille 2, 4 et $10 \mathrm{~mm}$. Ces classes sont retenues car elles permettent une comparaison au référentiel expérimental d'objets redistribués par ruissellement (Lenoble 2005).

La fabrique des cailloux et des objets archéologiques a été définie à partir de la mesure de la pente et de l'orientation de leur grand axe (au minimum 40 mesures par échantillon). Seuls les éléments dont le taux d'allongement $(\mathrm{a} / \mathrm{b})$ est supérieur à 2 , ont été pris en considération. Les séries de données ainsi obtenues ont été traitées selon deux méthodes statistiques (Bertran et Lenoble 2002). La première, de type bidimensionnel (Curray 1956), permet de définir un taux d'orientation L («Vector magnitude»), également utilisé pour calculer la probabilité $p$ d'obtenir une valeur plus élevée que $L$ par la combinaison de $n$ vecteurs d'orientation aléatoire (test de Rayleigh). La seconde, de type tridimensionnelle (Watson 1956 ; Mark 1973 ; Benn 1994), se base sur le calcul des valeurs propres normalisées E1, E2 et E3. Ces valeurs permettent d'établir les indices d'élongation EL $(=1-E 2 / E-1)$ et d'isotropie IS (=E3/E1) qui décrivent le degré d'organisation et la forme des fabriques (ceinture vs groupement pour le premier et ceinture ou groupement vs nuage pour le second). Nous avons eu recours au logiciel «Stereo » (McEachran 1990) pour le calcul des valeurs impliquées dans l'analyse tridimensionnelle des données.

Enfin, ces analyses ont été complétées par l'étude de l'organisation microscopique des sédiments. Celles-ci ont été réalisées à partir de lames minces de grandes dimensions taillées dans des blocs orientés, imprégnés sous vide par une résine polyester selon la technique préconisée par Guilloré (1980). La terminologie utilisée pour la description de ces lames est adaptée de celle définie par Bullock et alii (1985).

Les faciès ainsi caractérisés ont été interprétés par référence aux modèles sédimentaires définis en milieu actif.

\section{2 - Cadre géomorphologique du site}

Le site d'Isturitz correspond à une cavité de très grandes dimensions creusée dans un massif de calcaires urgoniens à faciès micritique et biopelsparitique (Boissonnas et al. 
1974). Ce massif barre la vallée de l'Arberoue dont le tracé est visiblement contrôlé par une structure chevauchante, orientée nord-sud (fig. 1).

La cavité d'Isturitz constitue l'étage supérieur d'un réseau karstique comportant trois niveaux, les étages moyen et inférieur étant dénommés respectivement Oxocelhaya et Erberua (fig. 2). Ces différents étages correspondent à d'anciens niveaux de base locaux et marquent donc des étapes de creusement de la vallée. Le réseau inférieur est actuellement emprunté par l'Arberoue.

Au nord-est du massif s'observe une ensellure (fig. 3) qui témoigne probablement d'un ancien passage de l'Arberoue. Etant donné son altitude (15 m au-dessus du fond de vallée, $45 \mathrm{~m}$ plus bas que l'entrée actuelle de la grotte d'Isturitz), ce passage a dû fonctionner jusqu'à une période assez tardive du Pléistocène.
Le massif karstique montre une nette dissymétrie des versants amont et aval. Le versant amont, orienté au sudest, est abrupt et convexe par suite du sapement de sa base par le cours d'eau. Le versant aval, orienté au nordouest, présente un profil concave par suite de la présence d'un prisme détritique à sa base.

Sur le versant amont (SE), se développe une langue de dépôts détritiques qui occupe un couloir de largeur décamétrique et s'étend de la partie sommitale du versant jusqu'à l'entrée actuelle de la grotte d'Isturitz. Ces dépôts, mis en place par ruissellement et par éboulisation, ont livré notamment du Moustérien. Ils sont partiellement cimentés par de la calcite et sont responsables de l'obturation de l'entrée sud-est de la cavité.

Sur le versant aval (NO), le prisme détritique mentionné plus haut a obturé l'entrée nord de la cavité et s'étend jusqu'à la base du versant. Ces dépôts, mis en place par

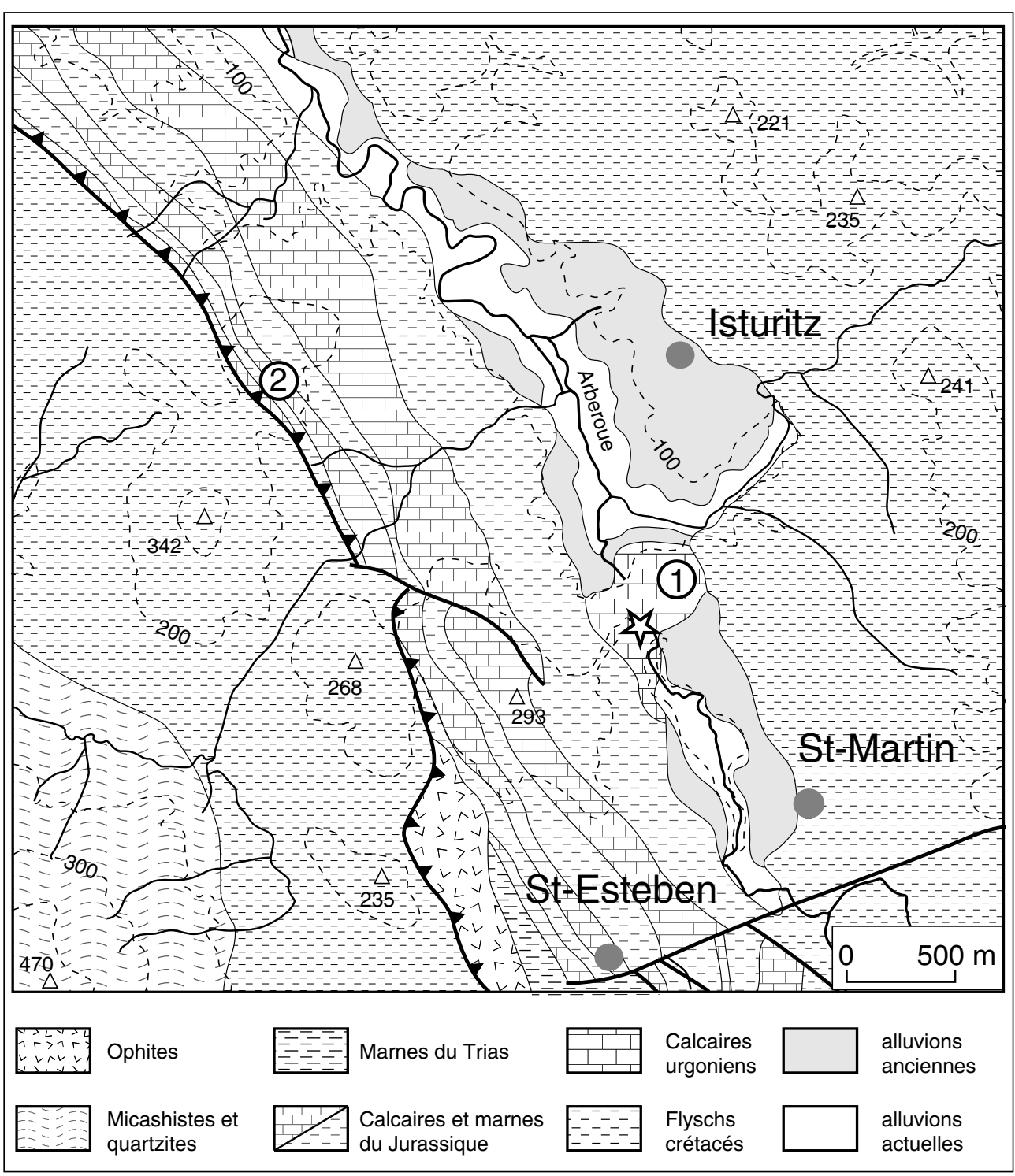

Figure 1 - Extrait de la carte géologique à 1/50 0000 d'Iholdy, modifié, montrant (1) la localisation du massif calcaire dans lequel s'ouvre la grotte d'Isturitz (étoile) ainsi que (2) le chevauchement mentionné dans le texte.

Figure 1 - Extract of the 1/50 000 geological map of Iholdy (modified) showing 1) the location of the calcareous block in which the cave of Isturitz (star) is carved as well as 2) the thrust mentioned in the text. 


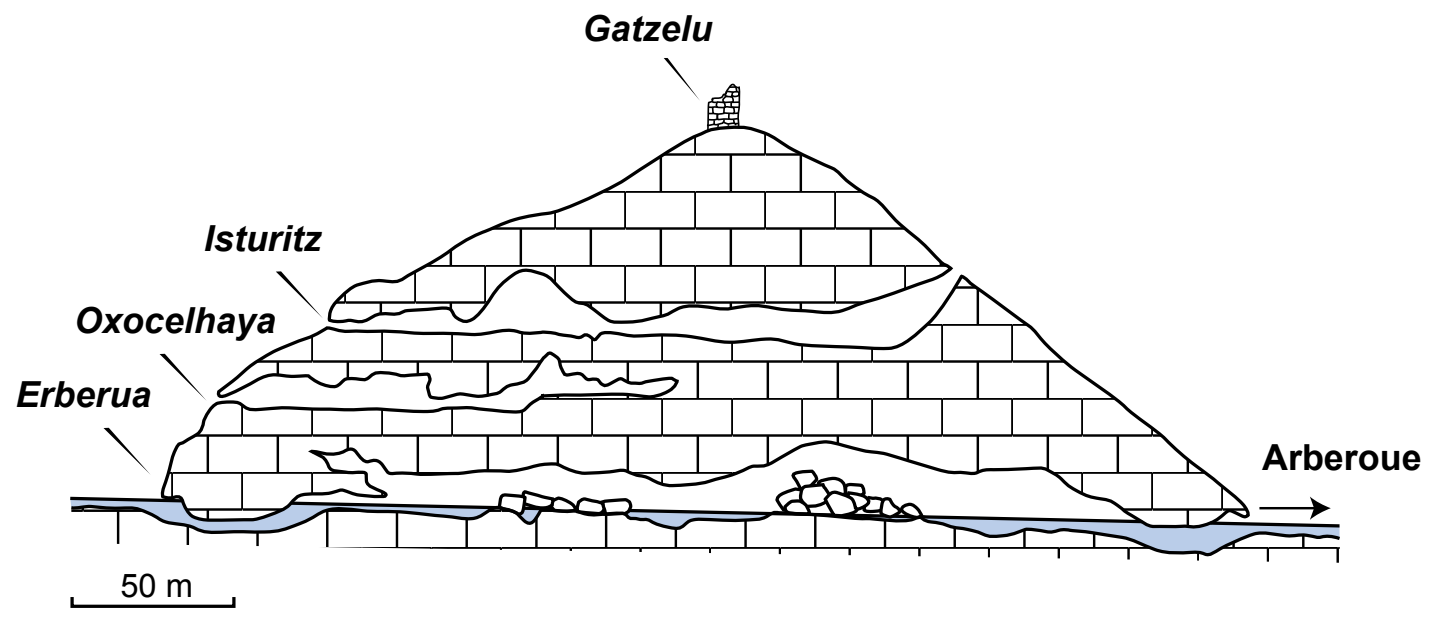

Figure 2 - Coupe SE-NO dans le massif calcaire montrant l'étagement des trois niveaux karstiques, selon Larribau et Prudhomme (1983, modifié). Figure 2 - SW-NE section of the calcareous hill showing the three stepped levels of the karstic system (following Larribau et Prudhomme, 1983, modified).

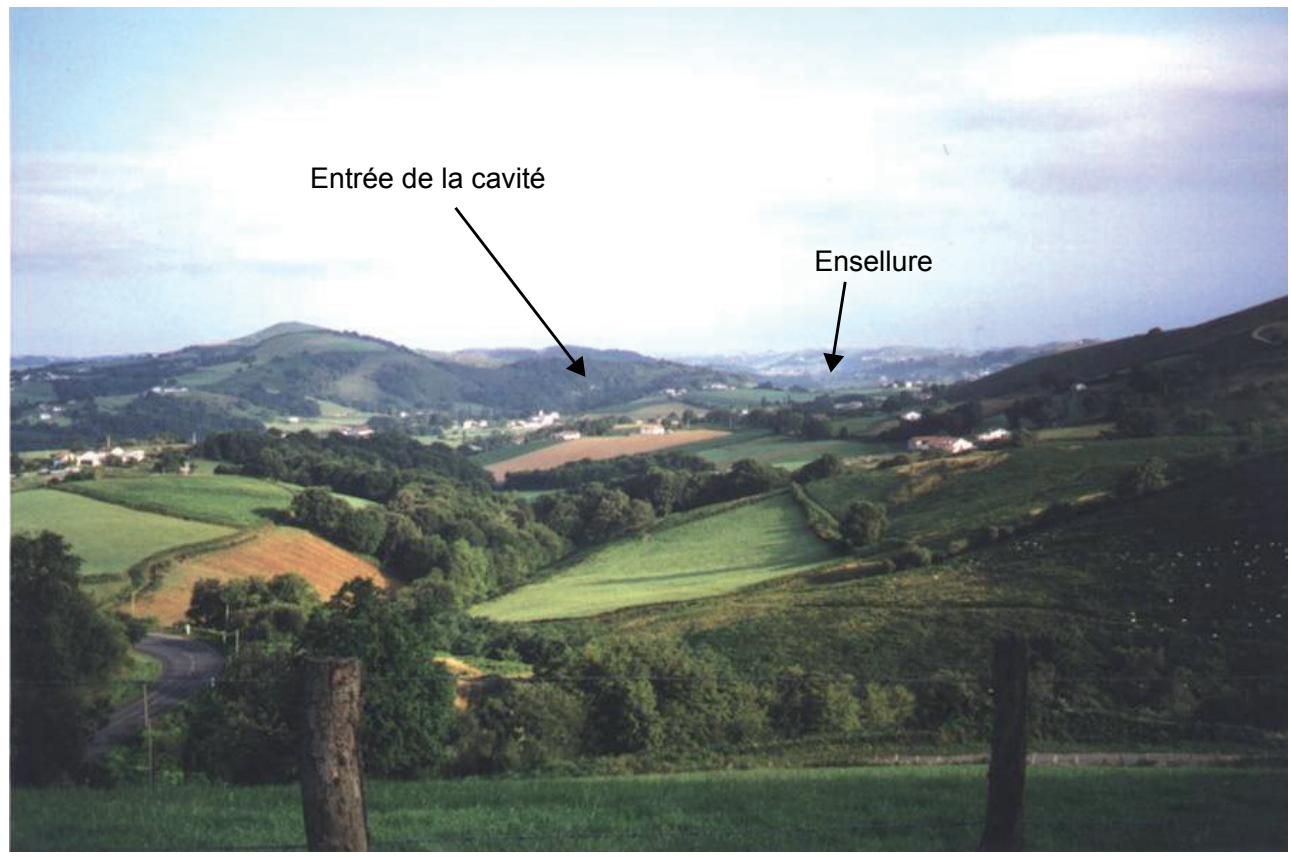

Figure 3 - Vue panoramique montrant l'ouverture actuelle de la cavité ainsi que l'ensellure qui, vers l'est, s'inscrit dans le massif calcaire.

Figure 3 - Panoramic view showing the present-day cave entrance as well as the morphological saddle that develops in the eastern part of the calcareous block. 
gravité, ont également pénétré dans la cavité jusqu'au niveau de l'actuel passage ménagé entre la grotte d'Isturitz stricto sensu et celle d'Oxocelhaya. Dans cette zone, ils ont livré des industries aurignaciennes, gravettiennes, solutréennes et magdaléniennes à R. et $\mathrm{S}$. Saint-Périer (Saint-Périer 1936 ; Saint-Périer et Saint-Périer 1952). L'accumulation a continué à se former au cours de l'Holocène puisque l'on retrouve des niveaux de l'Âge du Bronze enfouis à $3 \mathrm{~m}$ de profondeur (renseignement oral d'A. Turq). Cette accumulation a probablement, en partie au moins, une commande structurale : la roche-mère calcaire est découpée par des diaclases verticales à espacement décimétrique et d'orientation $\mathrm{N} 50^{\circ}$ à $\mathrm{N} 60^{\circ}$; celles-ci délimitent des panneaux rocheux qui s'ouvrent progressivement sous l'action de l'altération et de la pesanteur, puis basculent sur la pente en se fragmentant.

\section{3 - Évolution du réseau supérieur}

Les dépôts de remplissage ont été observés en de nombreux points de la grotte d'Isturitz, dans la salle StMartin comme dans la salle d'Isturitz (fig. 4). Les données ainsi recueillies permettent de proposer l'évolution globale suivante :

- Après une phase de creusement en contexte phréatique (Quaternaire ancien ?), se manifeste une longue évolution en contexte vadose : la cavité est d'abord traversée par un cours d'eau à régime torrentiel qui dépose les alluvions composées de très gros galets visibles dans la partie SO de la salle St-Martin ; puis, des percolations chargées en carbonates construisent d'imposants massifs stalagmitiques visibles notamment dans la salle d'Isturitz.

- Un écroulement important se produit dans la partie SO de la cavité. À la suite de celui-ci, ou de façon pénécontemporaine, il est possible que la cavité évolue momentanément en régime phréatique. Les poches de dissolution visibles sur les parois et sur le grand pilier stalagmitique de la salle d'Isturitz sont en effet classiquement interprétées comme le témoignage d'une action phréatique (Bretz 1942 ; White 1988). Cette hypothèse implique une remontée importante du niveau de base local et l'ennoiement de la cavité. Une telle évolution peut s'expliquer par un phénomène de type catastrophique, par exemple une avalanche rocheuse déclenchée à la suite d'un séisme et qui barre momentanément la vallée. Cette hypothèse, très plausible eu égard à la forte sismicité de la région considérée, permet également d'expliquer l'écroulement mentionné. Néanmoins, des chercheurs ont constaté que les déjections de chauvesouris pouvaient également produire des poches de dissolution (Lundberg et McFarlane 2009 ; Martini 2000). Cette seconde explication permet de faire «l'économie» d'une phase catastrophique mais ne rend pas compte de l'écroulement constaté.

- On observe ensuite un retour, ou la poursuite (selon l'hypothèse choisie), à une évolution en contexte vadose avec la formation de dépôts détritiques et phosphatés. D'une manière générale, la sédimentation évolue d'un pôle dominé par le ruissellement, voire par des écoulements de haute énergie liés au fonctionnement de ruisseaux souterrains, vers un pôle dominé par l'éboulisation. La dernière phase sédimentaire est de nature chimique et correspond à la formation d'un plancher stalagmitique, quasi-généralisé et daté de l'Holocène.

Cette évolution peut avoir des commandes à la fois intrinsèques (évolution du système karstique) et extrinsèques (évolution climatique régionale). En effet, la formation quasi-exclusive d'éboulis dans la partie sommitale des dépôts détritiques ainsi que l'épisode de sédimentation très réduite qui se place entre le dernier niveau d'Aurignacien et l'occupation magdalénienne sont probablement le reflet d'une phase froide à très froide. Celle-ci s'exprime également par le développement d'une structure lamellaire dans les dépôts à texture fine ainsi que par des phénomènes de cryoturbation (fig. 5) et de solifluxion affectant les couches renfermant les nappes de vestiges aurignaciens. Cette phase, probablement contemporaine d'un maximum de froid du stade isotopique 2, a pu occasionner le développement d'un pergélisol dont la présence est attestée ailleurs en Aquitaine (Texier et Bertran 1993 ; Texier 1996 ; Lenoble et al. 2012). Un tel phénomène pourrait avoir bloqué la plupart des infiltrations d'eau vers l'endokarst, induisant par la même occasion une relative paralysie du système. En effet, l'efficacité du gel est largement tributaire du degré de saturation de la roche-mère (e. g. Lautridou 1976 ; Berrisford 1991 ; Matsuoka 1991) et les transferts de matière (à l'état figuré ou dissout) sont très réduits par suite de la rareté et de l'incompétence des flux aqueux.

\section{4 - Étude géologique du secteur des fouilles conduites par $\mathrm{Ch}$. Normand}

\section{1 - Lithostratigraphie}

Cinq unités lithostratigraphiques ont été reconnues. Leur identification se base sur l'observation de la section dégagée par la fouille actuelle (fig. 6) et, pour les deux unités inférieures non encore atteintes par la fouille, par l'observation des coupes laissées par Passemard et Laplace. Les équivalences entre la lithostratigraphie, les subdivisions archéostratigraphiques de la fouille actuelle établies par A. Turq et C. Normand et les stratigraphies Passemard et Saint-Périer sont données dans le tableau 1.

On observe, de haut en bas :

- Unité I: Dépôt pluridécimétrique formé d'un plancher stalagmitique d'épaisseur variable dans lequel sont intercalées des lentilles d'argiles laminées brunes à brun gris très foncé $(2,5 Y \quad 3 / 2)$ épaisses de quelques centimètres. La limite inférieure de l'unité est nette. Les lentilles d'argiles livrent quelques vestiges pré- et protohistoriques.

- Unité II : Diamicton à support matriciel épais d'une dizaine de centimètres au sud évoluant vers un diamicton à support clastique d'épaisseur métrique au nord. Les blocs et cailloux calcaires sont anguleux et plutôt aplatis, très hétérométriques, non-orientés et parfois recouverts d'un enduit blanc crème phosphaté. 


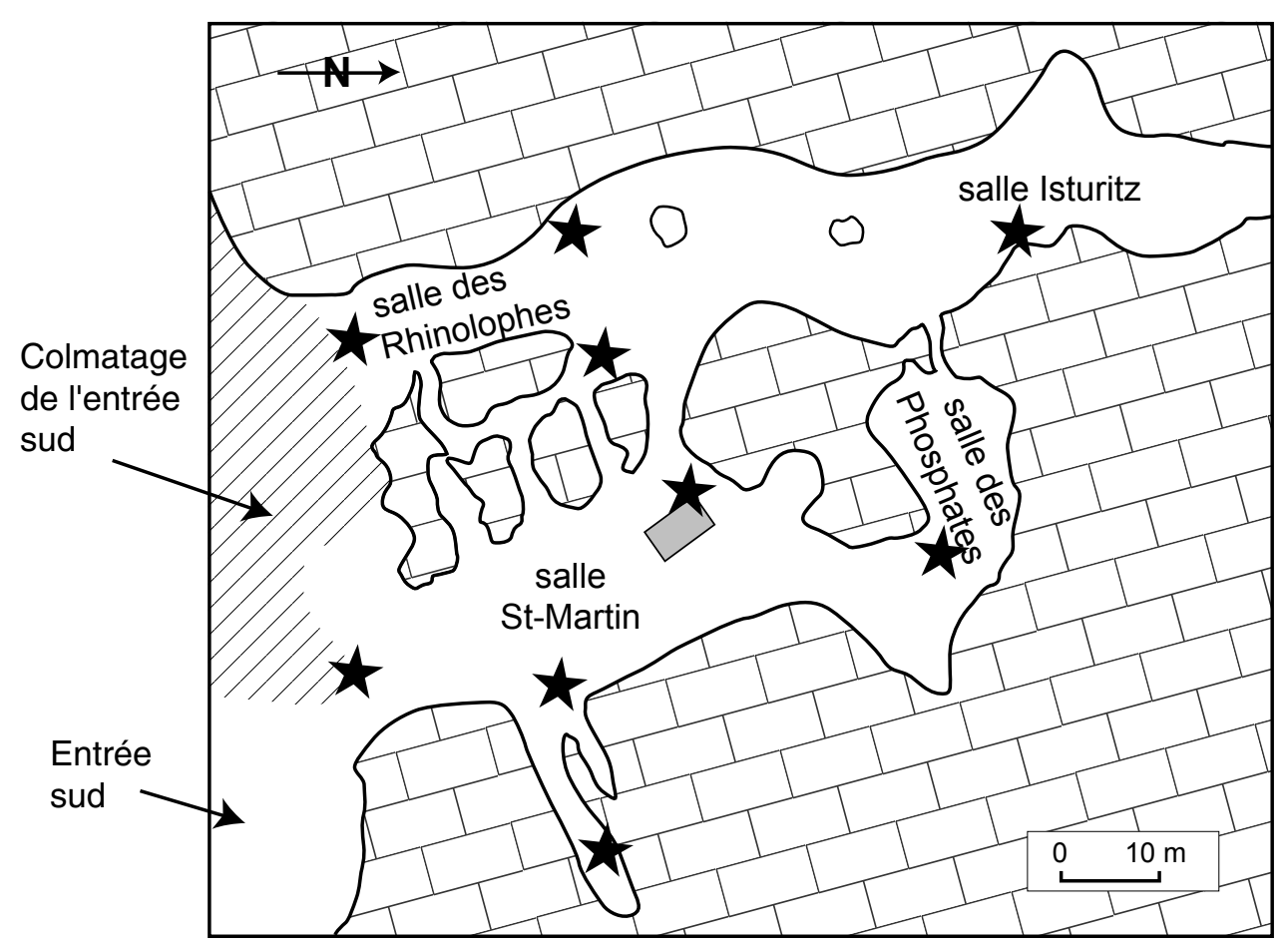

Figure 4 - Plan du niveau supérieur de la cavité. Les étoiles indiquent les points d'observation étudiés. Le rectangle gris représente la zone de fouille actuelle.

Figure 4 - Plan of the upper level of the karstic system. Stars indicate the study loci. The grey rectangle represents the current excavation zone.

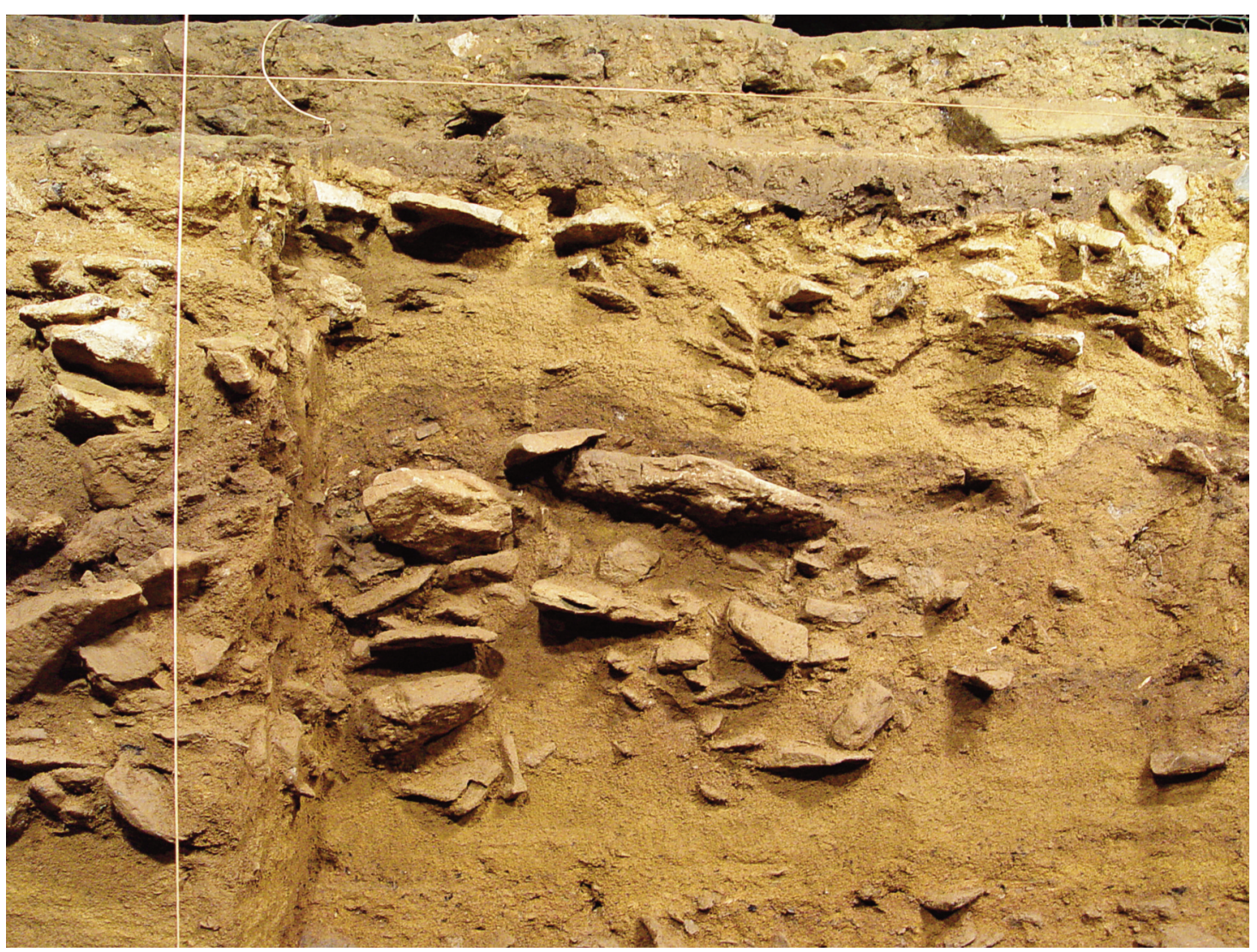

Figure 5 - Vue des involutions générées par cryoturbation. La puissance des dépôts photographiés est métrique. La vue est prise au niveau du carré V1 31 de la zone de fouille. Sur cette vue, on note la disposition variable des cailloux au sommet des dépôts, conformément aux déformations des contacts entre couches, ainsi que l'amortissement de ces déformations vers le bas de la section.

Figure 5 - View of cryoturbations. Pictured deposit is one-meter thick. This photo is taken in square V131 of the excavation area. Stones are lying in accordance with the distorted limits of units. Involutions tend to damp downwards. 


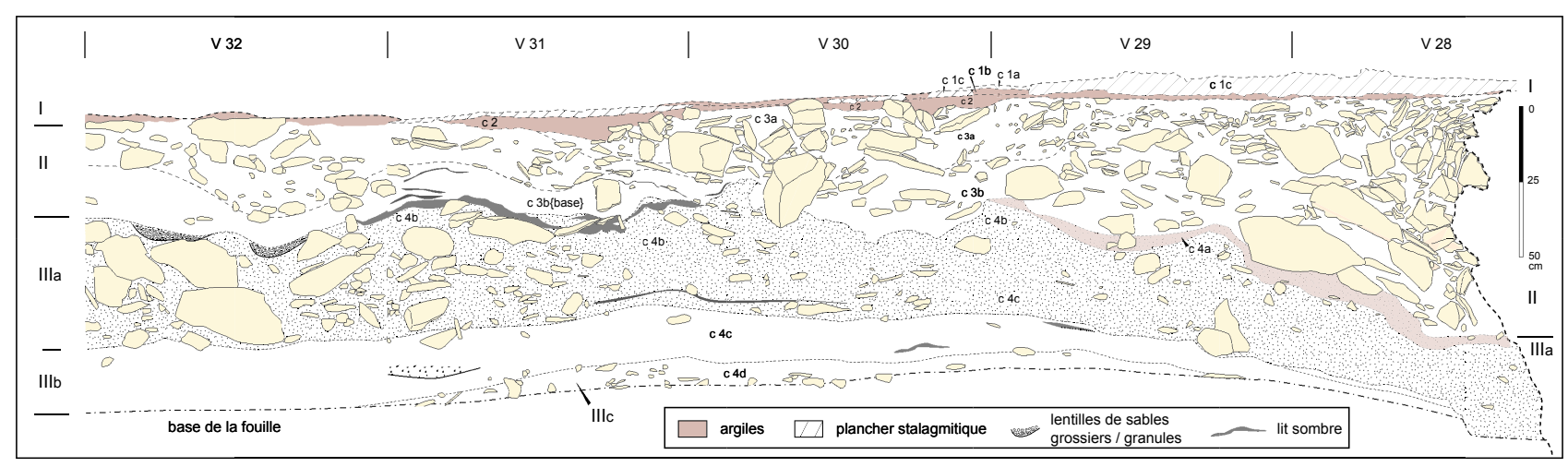

Figure 6 - Coupe ouest de la zone de fouille actuelle telle qu'elle pouvait être observée à la fin de la campagne 2005 (explication dans le texte).

Figure 6 - Section observed at the end of the 2005 campaign in the western part of the excavation zone (explanation in text).

Fouilles actuelles

Lithostratigraphie Archéostratigraphie

\begin{tabular}{|c|c|c|c|}
\hline Texier (1997) & (Normand, 2002) & (Passemard, 1944) & (Saint-Perrier R. et S., 1952) \\
\hline \multirow{2}{*}{ I } & c. $1(a, b$ et $c)$ & \multirow{2}{*}{ Couche stalagmitique } & \multirow{2}{*}{ Plancher } \\
\hline & c. 2 & & \\
\hline \multirow{3}{*}{ II } & \multirow{2}{*}{ Non représenté } & $E$ & SI \\
\hline & & $x, y$ & S II \\
\hline & c. $3 a$ et $b$ & \multirow{2}{*}{\multicolumn{2}{|c|}{ Non représenté }} \\
\hline \multirow{4}{*}{ III } & c. $3 b$ [base], c4a, & & \\
\hline & $c 4 b, c 4 c 1$ à c4c5 & \multirow{3}{*}{ A } & \multirow{3}{*}{ S III } \\
\hline & c. 4 c6 à c. 4 c12 & & \\
\hline & c. $4 \mathrm{~d} 1 \mathrm{a}$ & & \\
\hline IV & C. 5 & $M$ & \multirow{2}{*}{ S IV } \\
\hline V & c. 6 & Niveau à Ours & \\
\hline \multirow{2}{*}{\multicolumn{2}{|c|}{ - non observé - }} & \multirow[b]{2}{*}{ Limons à Ours } & Plancher \\
\hline & & & « sous-plancher » \\
\hline
\end{tabular}

Tableau 1 - Équivalences entre les différents découpages stratigraphiques.

Table 1 - Correspondence between the different stratigraphic systems.

La matrice est formée de sables limono-argileux brun vif (7,5YR 4/6) massifs. Elle présente une microstructure granulaire qui, en lames minces, prend l'apparence d'agrégats à coiffes enrobantes (fig. 7a).

Les variations de faciès de l'unité sont en relation avec la morphologie du plafond de la cavité. Ainsi, au sud de la zone de fouille, le dépôt n'est représenté que par des dalles plurimétriques liés à l'effondrement des bancs de

Fouilles anciennes

\section{Couches Passemard Couches Saint-Perrier}

(Saint-Perrier R. et S., 1952)

(1)




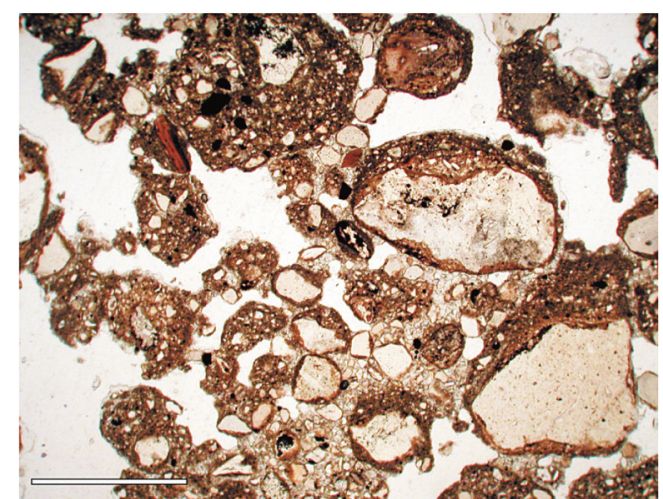

a

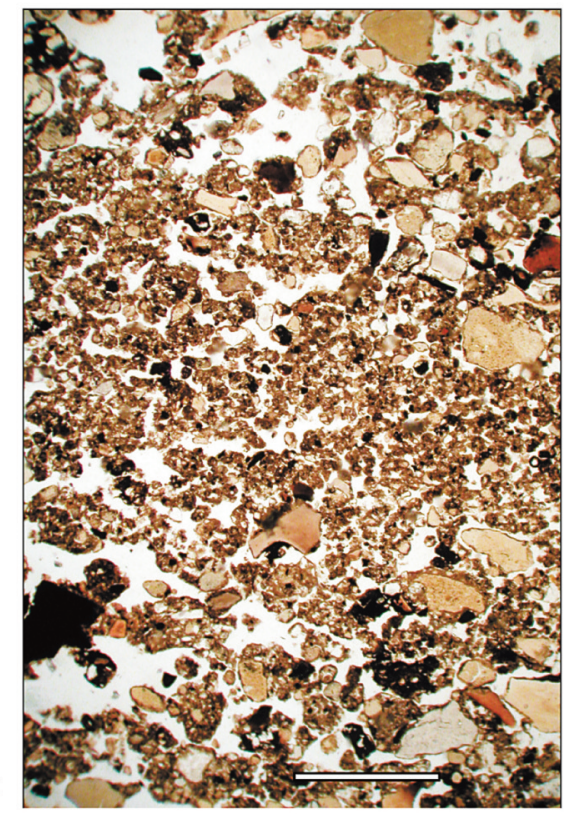

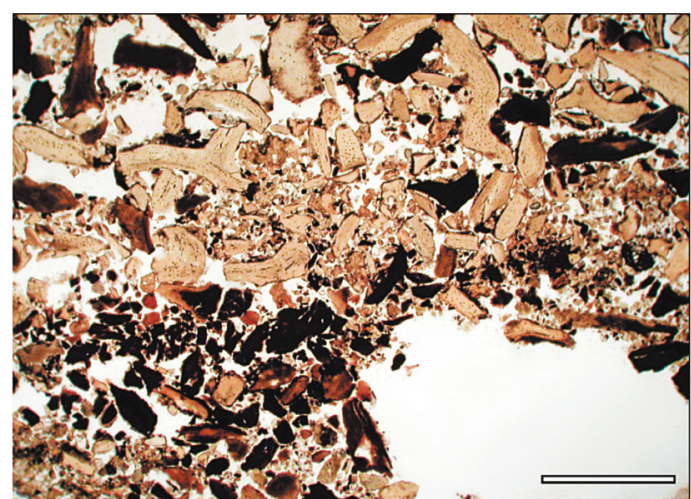

b

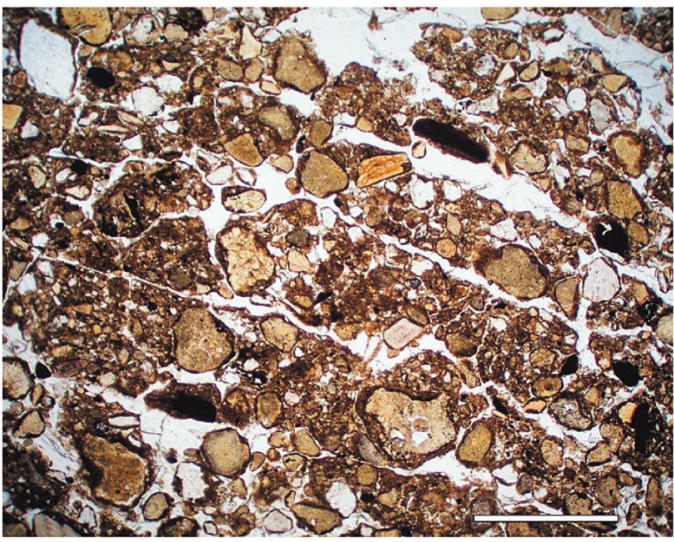

d

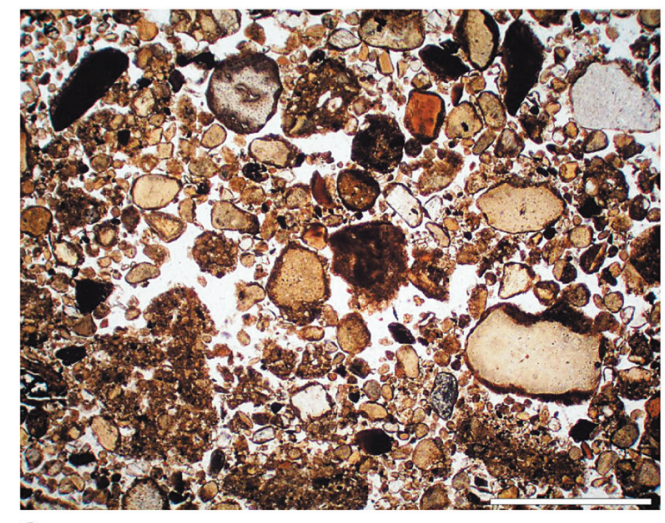

e

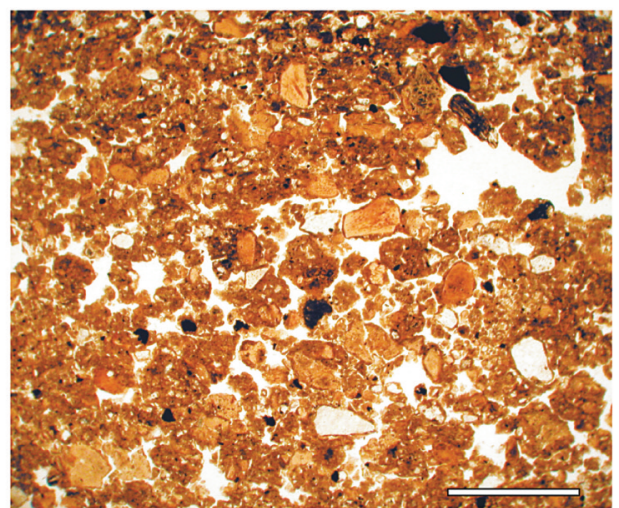

f

Figure 7 - Microfaciès des unités lithostratigraphiques II à IV : a, unité II, microstructure agrégée de la matrice. Les agrégats présentent des coiffes périphériques bien développées ( $L N$; trait : $1 \mathrm{~mm})$; $b$, unité Illa, détail d'une lentille riche en fraction archéologique : lamines d'os carbonisés et non brûlés triés (LN ; trait : $1 \mathrm{~mm}) ; c$, unité IIlc, vue partielle d'une figure de tri respectée par la structure lamellaire (LN ; trait : $1 \mathrm{~mm})$; d, unité IIlc, microstructure lamellaire superposée à une microstructure granulaire à nombreux ovoïdes (LN; trait : $1 \mathrm{~mm}) ; e$, unité IIIc, plage de sédiment éluvié. Des ovoïdes, plus ou moins désagrégés, voisinent avec des zones silteuses lavées (LN ; trait : 500 microns) ; $f$, unité $I V$, sommet, faciès de pseudo-sables et granules phosphatés ( $L N$; trait : $1 \mathrm{~mm}$ ).

Figure 7 - Microfacies of lithostratigraphic units II to IV: a, unit II; the matrix displays an aggregated microstructure. Well-developed cappings are visible around aggregates ( $L N$ - line: $1 \mathrm{~mm}) ; b$, unit IIlb. Detail of a sedimentary lens containing numerous archaeological remains. Laminae composed of sorted, carbonized and non-carbonized osseous fragments are visible (LN - line: $1 \mathrm{~mm})$; c, unit IIla; partial view of a sorted structure not affected by the platy structure $(L N$ - line: $1 \mathrm{~mm})$; $d$, unit III; a platy structure superimposed on a granular structure with numerous ovoids ( $L N$ - line: $1 \mathrm{~mm}$ ); e, unit IIlc; view of an eluviated zone. More or less disaggregated ovoids adjoin washed silty zones (LN - line: 500 microns); $f$, unit IV, upper part; pseudo-sands and phosphatic grains (LN - line: $1 \mathrm{~mm})$. 
inférieure est plus ou moins nette en fonction du contraste de couleur avec les dépôts sous-jacents. Cette limite est elle-même ondulée, conformément aux involutions présentes au sein de l'unité.

L'industrie magdalénienne rencontrée par Passemard (couche E) et R. et S. de Saint-Périer (couche S I) n'est pas représentée à l'emplacement des fouilles. Les vestiges recueillis dans cette unité sont rattachés à un aurignacien, " appartenant globalement à un faciès proche de l'Aurignacien ancien » (Normand 2002 - p. 98).

- Unité III : II s'agit d'un dépôt à stratification rudimentaire, épais en moyenne d'un mètre, qui contient une industrie proto-aurignacienne. La stratification du dépôt est liée à des variations de couleur et d'abondance en cailloux. Trois niveaux, dénommés de haut en bas IIIa, IIIb et IIIc, ont été distingués.

\section{Niveau IIla}

Ce niveau, épais de 30 à $40 \mathrm{~cm}$, présente une inclinaison modérée en direction du nord. II s'agit d'un diamicton à support clastique au sud s'appauvrissant progressivement en cailloux vers le nord. La fraction fine est formée de pseudo-sables limono-argileux dont la couleur varie du brun rouge foncé (5YR $2,5 / 2)$ au brun foncé $(7,5 Y R$ 4/4). La limite inférieure est nette, légèrement ondulée. On note une évolution latérale du faciès sédimentaire. Dans la partie sud du témoin, seules quelques petites structures infradécimétriques à base concave, associées à des sables grossiers et de petits granules, sont présentes au sommet de l'unité. Dans la partie médiane du témoin se développe une lamination grossière subhorizontale. À l'extrémité nord du témoin, elle laisse place à un dépôt lité. Le litage est alors formé de l'emboîtement de lentilles à morphologie en berceau, plus ou moins riches en granules et inclinées d'une dizaine de degrés vers le nord. Certaines de ces lentilles sont très riches en composantes archéologiques, principalement des esquilles d'os, brûlées ou non (fig. 7b).

En outre, au nord du témoin, la partie sommitale de ce niveau est marquée par un lit plus argileux, ocre rouge, de morphologie ondulée et d'épaisseur décimétrique.

\section{Niveau IIIb}

II s'agit d'un niveau lenticulaire d'extension plurimétrique épais d'une quinzaine de centimètres. II est formé de sables limono-argileux massifs brun à brun foncé $(7,5 Y R$ 4/4) et se distingue des niveaux sus- et sous-jacents par sa pauvreté en cailloux. Sa structure est massive, à l'exception de quelques lits isolés, plus foncés ou plus sableux. La limite inférieure est nette, légèrement ondulée, soulignée par des petits granules et graviers. Ce contact est subhorizontal, hormis à l'extrémité nord du témoin où son inclinaison atteint une vingtaine de degré.

\section{Niveau IIIc}

Seul le sommet de ce niveau a été observé. Il est formé d'un cailloutis associé à une matrice de sables argilo-limoneux brun rouge foncé (5YR 3/3). La fouille a montré que ce cailloutis correspond à un pavage résiduel, composé de fragments rocheux juxtaposés de taille variée (fig. 8). Les pièces archéologiques associées sont imbriquées entre les débris calcaires et présentent une orientation préférentielle associant deux modes; l'un conforme à la pente, l'autre transverse à celle-ci.

En lames minces, les pseudo-sables apparaissent formés d'agrégats émoussés fréquemment enrobés d'une coiffe à texture fine. Les traits sédimentaires sont rares (fig. 7c). Le microfaciès dominant est celui d'une microstructure lamellaire se surimposant à la structure granulaire originelle du dépôt (fig. 7d). On observe également des plages éluviées qui se caractérisent par un appauvrissement en fraction fine du fond matriciel et des coiffes qui enrobent les agrégats. Ce phénomène est particulièrement visible au niveau du pavage du niveau Illc (fig. 7e).

\section{- Unité IV :}

Dépôt épais de 30 à $60 \mathrm{~cm}$, formé de limons argileux jaunes massifs ou à débit polyédrique et à sous-structure granulaire plus ou moins nette. Sous le microscope, les sédiments apparaissent formés de l'entassement d'agrégats arrondis comportant fréquemment un noyau phosphaté (fig. 7f). On observe localement une structure lamellaire. Cette unité contient quelques cailloux dispersés présentant parfois un cortex d'altération développé. Sa limite inférieure est nette.

- Unité V (épaisseur non reconnue) :

Dépôt caillouteux et graveleux à structure ouverte à semiouverte contenant localement des lentilles de matériau trié. Les clastes sont plus ou moins émoussés.

\section{2 - Genèse des dépôts}

La mise en place des dépôts de la Salle Saint-Martin peut être résumée comme suit :

- L'organisation litée, l'émoussé des clastes et la texture grossière indiquent que les dépôts de l'unité $\mathrm{V}$ résultent d'écoulements turbulents à forte compétence.

- L'unité IV correspond à une accumulation de pseudosables transportés par ruissellement. Le tri et l'émoussé des grains en témoignent. Un gel contemporain du sol a probablement favorisé la production d'agrégats (Bertran 1999). Les coiffes matricielles qui enrobent les grains caractérisent en effet les agrégats produits dans les horizons superficiels des cryosols (Van Vliet-Lanoë 1988). Ces granules proviennent de l'érosion de dépôts préexistants de la cavité, comme l'indiquent leur nature phosphatée. La présence de petites esquilles osseuses dans certains d'entre eux indiquent que ces derniers proviennent de la fragmentation de coprolithes (Horwitz et Goldberg 1989).

- Le ruissellement, l'éboulisation et la solifluxion ont contribué dans des proportions variables à l'édification des dépôts des unités II et III. Les petites structures à base concave comblées d'un sédiment grossier représentent des comblements de rigoles (Lenoble 2005). Celles-ci, ajoutées à la lamination du sédiment, attestent de l'action dominante du ruissellement concentré dans l'apport des matériaux fins. En outre, la présence d'un litage en relation avec des structures érosives (emboîtement de lentilles, lits 


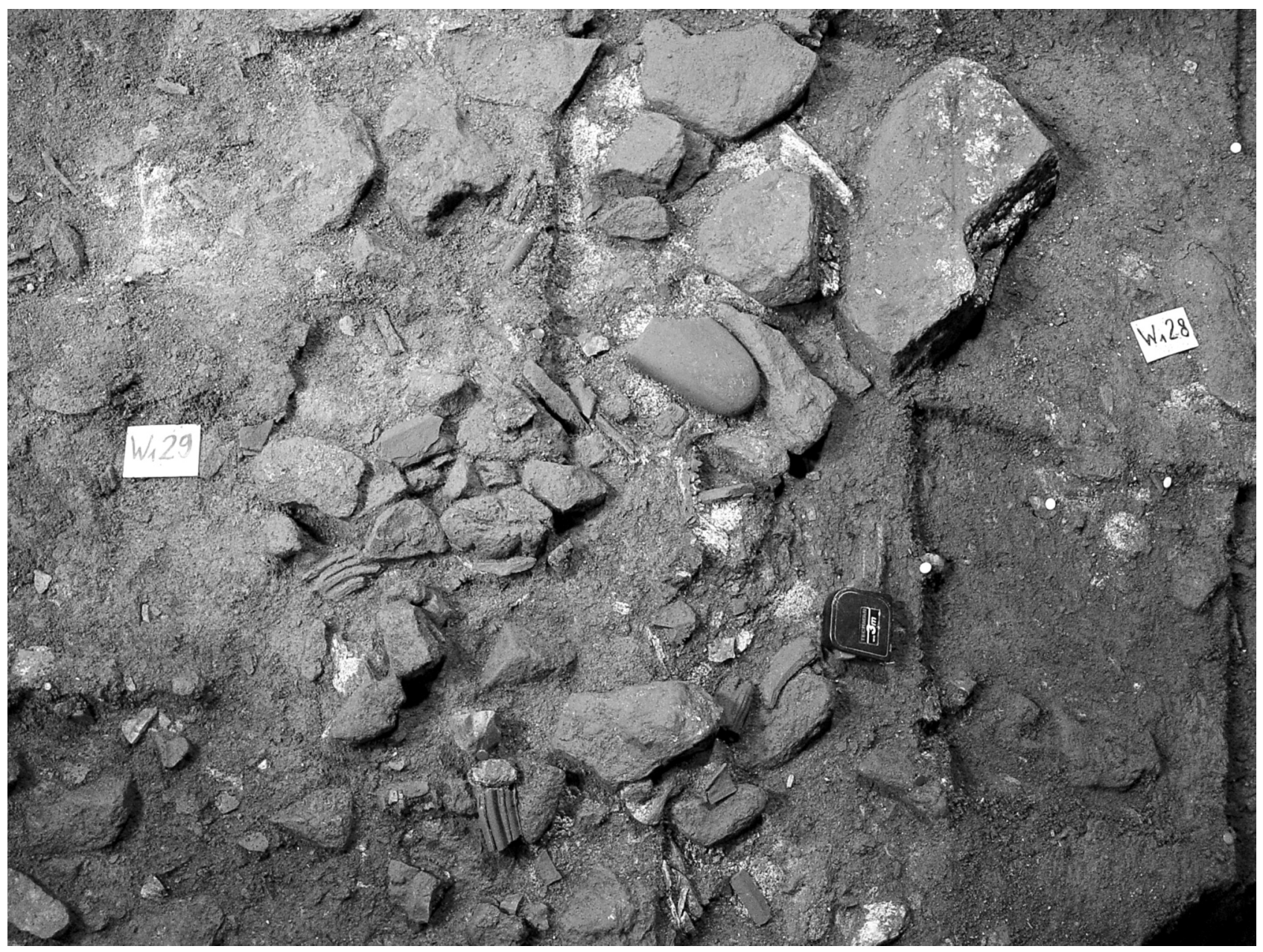

Figure 8 - Vue du pavage résiduel présent au sommet de l'unité IIlc.

Figure 8 - View of the runoff pavement located in the upper part of unit IIIc.

de granules, pavages résiduel) dans le secteur nord du témoin indique, qu'à cet emplacement, les écoulements étaient très compétents. La production gravitaire est importante, voire prédominante, au sommet de l'unité III et au sein de l'unité II. Outre les caractéristiques de la fraction caillouteuse (absence de tri, fabrique isotrope), la relation étroite entre l'importance et la nature des blocs et cailloux d'une part, et la morphologie du plafond de la cavité d'autre part, atteste d'une simple chute des débris détachés des parois.

- De nombreux ovoïdes sont présents parmi les pseudosables de l'unité III. Ils indiquent, là aussi, qu'un gel du sol s'est manifesté lors de la formation du dépôt. Qui plus est, l'observation de blocs morcelés aux fragments déplacés, de lamines ondulées et d'une microstructure lamellaire associée indique une retouche secondaire des dépôts par solifluxion. Cette dynamique n'a toutefois pas profondément remanié le dépôt dans la mesure où les structures sédimentaires originelles sont encore présentes.
- Un cryosol se développe au sommet de l'unité II. En effet, l'espacement régulier des involutions qui affectent cette unité et leur association à une microstructure ovoïde bien développée montre qu'on est en présence d'un phénomène de cryoturbation. La genèse de ce sol fossile implique une stabilité morphologique locale. Cet arrêt de sédimentation est également attesté par l'altération des débris calcaires depuis le sommet de l'unité et liée à la redistribution de phosphates à l'origine des pellicules enrobant les débris (Campy et Chaline 1993). Par ailleurs, la formation de ce cryosol implique un climat plus froid que l'actuel ; on retrouve ici l'empreinte d'un épisode très froid du stade 2 observé dans l'ensemble du réseau supérieur (cf. supra, paragraphe «Evolution du réseau supérieur»). - L'unité I correspond essentiellement à l'édification d'un plancher stalagmitique. La sédimentation détritique se limite à des dépôts de décantation dans des flaques. Ce changement radical de style de sédimentation conduit à rapporter cette unité à l'Holocène. 


\section{5 - Mise en place des nappes de vestiges aurignaciennes}

Les unités II et III livrent en séquence des industries se rapprochant de l'Aurignacien ancien d'une part, et du protoAurignacien, d'autre part (Normand et al. 2007). La puissance de l'unité III et la richesse du matériel archéologique qu'elle contient laissent espérer l'identification de plusieurs stades évolutifs au sein du proto-Aurignacien (Szmidt et al. 2010). Pour ces deux raisons, la séquence du site est unique et remarquable.

Cependant, avant toute interprétation, il convient de s'interroger sur l'origine strictement culturelle de la distribution stratigraphique des vestiges. En effet, comme le montre l'étude géologique, les niveaux archéologiques sont associés à des dynamiques sédimentaires variées : éboulisation, ruissellement et solifluxion. La question d'une modification de l'organisation des vestiges par ces agents naturels se pose donc. C'est pour y répondre que l'étude de la mise en place des nappes de vestiges a été entreprise.

Ce travail prend appui sur l'étude sédimentologique. II ressort de celle-ci que le ruissellement concentré est le principal agent de sédimentation de l'unité III. L'éboulisation et la solifluxion ont également participé à l'édification des dépôts, mais l'étude sédimentologique indique que leur contribution est plus modeste (cf. supra). C'est pourquoi l'étude de la formation du site s'est surtout focalisée sur l'impact qu'a pu avoir le ruissellement dans la constitution des nappes de vestiges.

La démarche consiste alors à rechercher au sein des séries archéologiques les caractéristiques propres à cet agent naturel. Quatre critères sont utilisés à cette fin (Lenoble 2005). Ce sont :

1 - les organisations remarquables, c'est-à-dire des structures sédimentaires mettant en jeu des vestiges archéologiques ;

2 - la distribution verticale des vestiges;

3 - les tris granulométriques du matériel archéologique ;

4 - la fabrique des objets.

Les lustres d'abrasion n'ont pas été pris en compte car les vestiges sont patinés ultérieurement à leur enfouissement.
Des remontages ont été réalisés (Normand 2002), mais ils ont été jugés trop peu nombreux pour tenter d'en tirer profit.

\section{1 - Données analytiques}

\section{Organisations remarquables}

Le pavage résiduel contenu dans l'unité Illc (cf. supra) constitue une organisation remarquable. Comme le montre la figure 8, des objets archéologiques de taille importante, dents et silex taillés de 3 à $5 \mathrm{~cm}$, sont bloqués entre les cailloux du pavage. L'orientation de ces objets, conforme ou perpendiculaire à la direction de la pente, indique qu'ils ont été piégés lors de leur transport. En milieu de ruissellement, ces pavages se forment dans la zone d'érosion ou de transit et les objets bloqués représentent les plus gros objets immobilisés au cours du transport (Lenoble 2005). On peut donc en déduire :

1 - que les objets qui sont associés au pavage sont en position secondaire,

2 - que les objets plus petits, soit la plus grande partie des ensembles archéologiques, ont été transportés au-delà et, par voie de conséquence, que la série contenue dans ce niveau Illc ne représente qu'une petite partie de l'ensemble originel de vestiges.

\section{Fabriques}

La fabrique des vestiges de l'unité III a été mesurée en deux endroits de la fouille : l'une au sud, dans le faciès de diamicton à support clastique (carré $\mathrm{W}_{1} 32$ ) et l'autre au nord, au sein des pseudo-sables lités (carrés $V_{1} 28$ et $V_{1} 29$ ). La fabrique des cailloux du diamicton a également été mesurée (tabl. 2).

Ces mesures sont confrontées au référentiel expérimental par report sur le diagramme de Benn. Comme le montre la figure 9 , les mesures réalisées dans le faciès diamictique de l'unité IIla ne sont pas compatibles avec une redistribution des vestiges par ruissellement, mais sont semblables à celles d'éboulis gravitaire, à l'image de la série de cailloux mesurés. Sur ce même diagramme, il apparaît que la fabrique des objets contenus dans le faciès

\begin{tabular}{|c|c|c|c|c|c|c|c|c|c|}
\hline \multirow{2}{*}{ Série } & \multirow[t]{2}{*}{$N$} & \multirow{2}{*}{ Années } & \multicolumn{2}{|c|}{ Test de Curray } & \multicolumn{3}{|c|}{$\begin{array}{l}\text { Vecteurs propres } \\
\text { normalisés }\end{array}$} & \multicolumn{2}{|c|}{$\begin{array}{l}\text { Indices de } \\
\text { Benn }\end{array}$} \\
\hline & & & $L$ & $p$ & E1 & E2 & E3 & $E L$ & IS \\
\hline \multicolumn{10}{|c|}{$W_{1} 31$ (diamicton à support clastique) } \\
\hline vestiges & 38 & 2000-2002 & 18,0 & 0,311 & 0,503 & 0,325 & 0,172 & 0,354 & 0,342 \\
\hline cailloux & 36 & $2000-2002$ & 13,1 & 0,540 & 0,511 & 0,367 & 0,122 & 0,282 & 0,239 \\
\hline \multicolumn{10}{|c|}{$V_{1} 28 \& 29$ (pseudo-sables lités) } \\
\hline vestiges & 45 & $2000-2002$ & 10,7 & 0,599 & 0,510 & 0,417 & 0,072 & 0,182 & 0,141 \\
\hline vestiges & 101 & 2004-2005 & 2,9 & 0,920 & 0,487 & 0,448 & 0,065 & 0,080 & 0,133 \\
\hline
\end{tabular}

Tableau 2 - Caractérisation statistique des fabriques de vestiges archéologiques et cailloux de l'unité III.

Table 2 - Statistical characterization of fabrics of archaeological remains and rock fragments contained in unit III. 


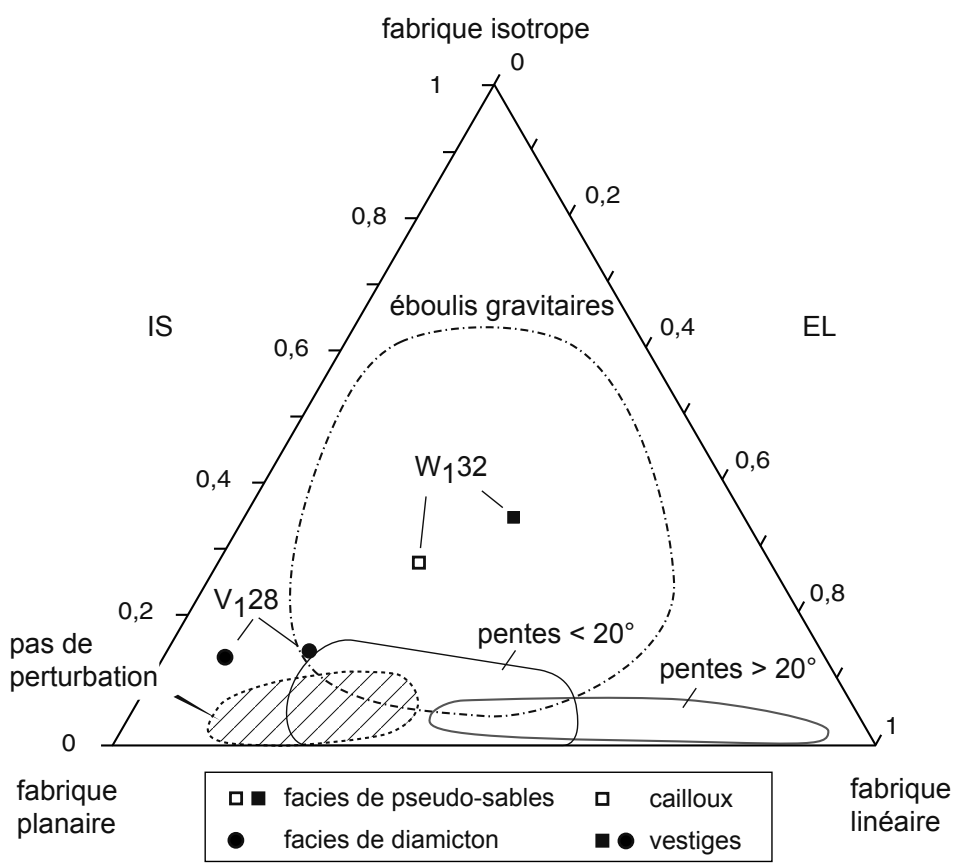

Figure 9 - Fabrique des vestiges et cailloux de l'unité III, diagramme de Benn. Les courbes enveloppes des fabriques de ruissellement, d'éboulis gravitaires et de sites archéologiques non perturbés sont également reportées, selon Bertran et Lenoble (2002).

Figure 9 - Benn diagram showing the position of fabrics measured on archaeological pieces and rock fragments of unit III compared with areas of runoff, rockfall and undisturbed archaeological assemblages as defined by Bertran and Lenoble (2002). de pseudo-sables lités de l'unité III n'est pas diagnostique. Les deux séries de mesures réalisées se rapprochent des objets déplacés par ruissellement tout autant que des sites non perturbés.

Le grand nombre de mesures faites au cours des années 2004 et $2005(N=101)$ permet d'approfondir ces résultats. Pour cela, les paramètres L et $p$ de Curray (1956) ont été établis sur des séries de 30 objets se succédant dans l'ordre stratigraphique (fig. 10A). Cette figure montre des variations importantes, ce qui est attendu pour des dépôts de ruissellement (Bertran et Lenoble 2002). Le même graphique est établi avec la valeur doublée des angles (fig. 10B). Cette procédure est utilisée dans le cas d'une distribution bimodale des orientations. Elle a pour effet de superposer des modes initialement transverse (Baschelet 1981). C'est pourquoi, si la série est bimodale, l'intensité d'orientation $\mathrm{L}$ augmente lorsque les angles sont doublés. La figure 10B montre que ce cas de figure est fréquent au sein des dépôts de l'unité III d'Isturitz et que, dans certains cas, l'orientation préférentielle des objets atteint des valeurs qui excluent un effet d'échantillonnage $(p<0,05)$. En milieu de ruissellement, une distribution bimodale des objets s'observe dans le cas de matériel redistribué en rigoles (Lenoble 2005). C'est pourquoi les zones grisées de la figure 10B sont interprétées comme des passées de matériel redistribué par ruissellement.

On peut ainsi déduire de ces différents traitements statistiques :

1 - que la nappe de vestiges contenue dans le faciès de pseudo-sables lités IIla et IIIb en dehors des secteurs à faciès gravitaire n'est pas homogène ;

2 - qu'elle est constituée par l'alternance de passées où la fabrique indique une redistribution des vestiges et de passées où la fabrique n'est pas diagnostique ;

3 - que cette redistribution est le fait du ruissellement.

\section{Tri dimensionnel}

La recherche d'un tri granulométrique des séries archéologiques a été menée à partir du matériel récolté dans deux sous-carrés de $33 \times 16 \mathrm{~cm}$ au cours des années 2000 à 2002. Ces sous-carrés ont été sélectionnés de façon à ce que, en sus de l'unité II, les différents faciès de l'unité III soient représentés : diamicton à support clastique dans le sous-carré $V_{1} 31 \mathrm{~b}$ et pseudo-sables lités dans le sous-carré $V_{1} 29 \mathrm{~b}$. Les résultats sont portés dans le tableau 3. Ils sont comparés au référentiel expérimental des assemblages dimensionnels produits par débitage de roche dure (Bertran et al. 2006), d'une part, et à celui des tris de vestiges archéologiques affectés par ruissellement par l'intermédiaire du triangle des classes dimensionnelles (Lenoble 2005).

Deux observations principales s'en dégagent (fig. 11) : 1 - une distinction majeure apparaît entre les deux unités. Les vestiges de l'unité II sont systématiquement triés, soit par absence de fraction grossière $\left(V_{1} 29 b\right)$, soit, au contraire, par sous-représentation de fraction fine $\left(\mathrm{V}_{1} 31 \mathrm{~b}\right)$; 2 - les proportions des différentes classes dimensionnelles de vestiges de l'unité III suivent, pour la plupart, celles d'ensembles expérimentaux non triés, en accord avec le constat que la majeure partie des silex taillés recueillis à la fouille résultent d'une activité de débitage à même le site (Normand et al. 2007). S'en distinguent quelques décapages, parfois successifs, dans lesquels la petite fraction est sous-représentée.

L'interprétation qui peut en être faite est la suivante : - dans le cas de l'unité II, l'absence d'une partie de la petite fraction peut être imputée à la cimentation des dépôts. En revanche, la sous-représentation de la fraction de grande dimension $\left(\mathrm{V}_{1} 29\right.$, décapage 6-8 ou $\mathrm{V}_{1} 31$ décapage 6-9) 


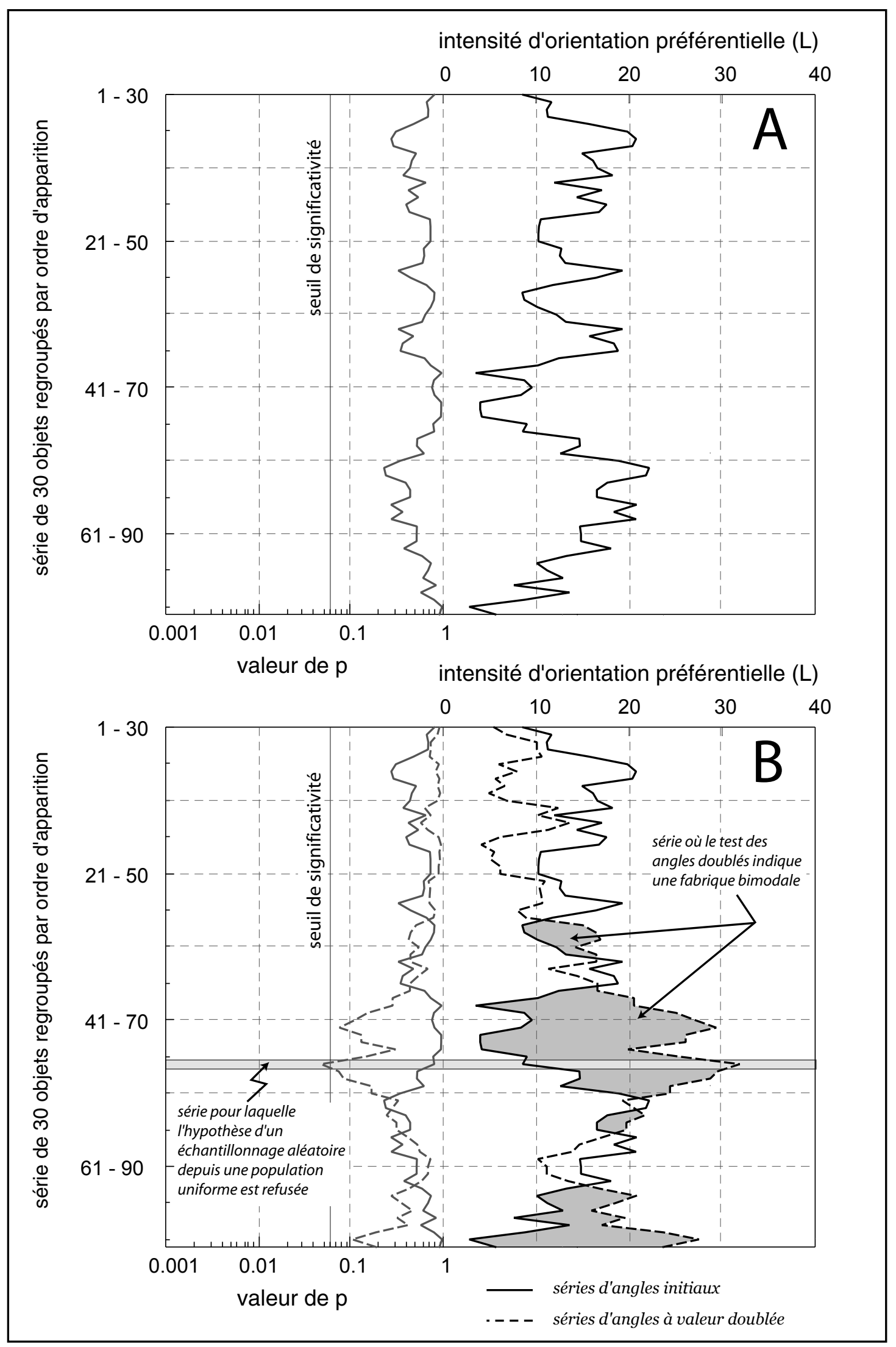

Figure 10 - A - évolution verticale du taux d'orientation (L). B - Comparaison entre l'évolution verticale du taux d'orientation calculé à partir des séries de mesures initiales et à partir des séries d'orientation à valeurs d'angles doublées. Les courbes représentent les valeurs glissantes établies sur des séries de 30 objets parmi les 101 pièces mesurées ordonnées selon leur ordre d'apparition dans le dépôt.

Figure 10 - A - Vertical evolution of the vector magnitude L. B - Comparison between the vertical evolution of the vector magnitude calculated from the series of initial orientation measurements and from the series with doubled angle values. Curves represent moving values based on series of thirty measures subset from the 101 measures put in order from the top to bottom. 


\begin{tabular}{|c|c|c|c|c|c|c|c|c|c|c|c|c|c|}
\hline Déc. & Prof & $>2$ & $>4$ & $>10$ & $\mathrm{~N}$ & Unité & Déc. & Prof & $>2$ & $>4$ & $>10$ & $\mathrm{~N}$ & Unité \\
\hline \multicolumn{14}{|c|}{ Sous-carré $V_{1} 31 b$} \\
\hline 6 & |14-20 & 0 & 100 & 0 & 1 & \multirow{10}{*}{ II } & 17 & $|36-40|$ & 56,0 & 40,0 & 4,0 & 75 & \multirow{11}{*}{ III } \\
\hline 7 & $22-24$ & 80,0 & 20,0 & 0 & 10 & & 18 & $40-42$ & 62,2 & 32,9 & 4,9 & 82 & \\
\hline 8 & $24-26$ & 42,9 & 57,1 & 0 & 7 & & 19 & $42-44$ & 36,1 & 55,6 & 8,3 & 24 & \\
\hline 9 & $26-30$ & 66,7 & 33,3 & 0 & 39 & & 20 & $44-46$ & 71,7 & 25,3 & 3,0 & 300 & \\
\hline 10 & $28-30$ & 31,3 & 68,8 & 0 & 16 & & 21 & $46-48$ & 53,2 & 41,5 & 5,3 & 94 & \\
\hline 11 & $30-32$ & 37,5 & 62,5 & 0 & 16 & & 22 & $49-52$ & 47,7 & 29,5 & 22,7 & 44 & \\
\hline 12 & $32-34$ & 52,8 & 38,9 & 8,3 & 36 & & 23 & $52-54$ & 62,4 & 33,3 & 4,3 & 93 & \\
\hline 13 & $34-36$ & 50,0 & 50,0 & 0 & 26 & & 24 & $54-56$ & 51,3 & 39,5 & 9,2 & 76 & \\
\hline 14 & $36-38$ & 28,0 & 60,0 & 12,0 & 25 & & 25 & $56-58$ & 48,5 & 37,9 & 13,6 & 66 & \\
\hline 15 & $38-40$ & 0 & 81,3 & 18,8 & 16 & & 26 & $58-60$ & 67,4 & 29,3 & 3,3 & 92 & \\
\hline 16 & $40-42$ & 84,8 & 15,2 & 0 & 46 & III & 27 & $60-64$ & 74,2 & 21,6 & 4,1 & 97 & \\
\hline \multicolumn{14}{|c|}{ Sous-carré $V_{1} 29 b$} \\
\hline 6 & | 22-24 & 50,0 & 50,0 & 0 & 4 & \multirow{6}{*}{$\|$} & 21 & |58-60 & 58,6 & 36,9 & 4,5 & 244 & \multirow{15}{*}{ III } \\
\hline 7 & $24-28$ & 25,0 & 66,7 & 8,3 & 12 & & 22 & $60-66$ & - & - & - & - & \\
\hline 8 & $29-32$ & 17,4 & 82,6 & 0,0 & 23 & & 23 & 64-66 & 23,6 & 60,7 & 15,7 & 89 & \\
\hline 9 & $32-36$ & 35,7 & 50,0 & 14,3 & 42 & & 24 & $66-68$ & 48,5 & 42,4 & 9,1 & 33 & \\
\hline 10 & $36-40$ & 38,2 & 52,7 & 9,1 & 55 & & 25 & $68-70$ & 63,4 & 33,2 & 3,4 & 262 & \\
\hline 11 & $40-46$ & & & & & & 26 & $70-72$ & 38,4 & 52,1 & 9,6 & 73 & \\
\hline 12 & $32-36$ & 45,1 & 53,7 & 1,2 & 82 & \multirow{9}{*}{ III } & 27 & $72-74$ & 53,0 & 43,6 & 3,4 & 117 & \\
\hline 13 & $40-50$ & 62,3 & 34,4 & 3,3 & 61 & & 28 & $74-76$ & 68,0 & 29,1 & 2,9 & 103 & \\
\hline 14 & $42-46$ & 61,7 & 28,3 & 10,0 & 60 & & 29 & $76-78$ & 35,3 & 64,7 & 0 & 17 & \\
\hline 15 & $46-48$ & 53,6 & 39,1 & 7,2 & 69 & & 30 & $78-80$ & 81,4 & 16,3 & 2,3 & 43 & \\
\hline 16 & $48-50$ & 50,8 & 39,7 & 9,5 & 63 & & 31 & 78-80 & 61,3 & 34,7 & 4,0 & 75 & \\
\hline 17 & $50-54$ & 60,2 & 34,4 & 5,4 & 349 & & 32 & $76-80$ & 61,0 & 30,5 & 8,5 & 82 & \\
\hline 18 & $54-56$ & 64,2 & 31,8 & 4,1 & 296 & & 33 & $80-84$ & 60,5 & 37,2 & 2,3 & 172 & \\
\hline 19 & $52-56$ & 68,8 & 27,3 & 3,9 & 128 & & 34 & 84-88 & 64,8 & 29,6 & 5,6 & 54 & \\
\hline 20 & $56-58$ & 64,2 & 31,6 & 4,2 & 212 & & & & & & & & \\
\hline
\end{tabular}

Tableau 3 - Isturitz, salle Saint-Martin. Proportions des vestiges lithiques par classes dimensionnelles pour les deux colonnes-échantillon. Les classes dimensionnelles sont exprimées en largeur de maille $(\mathrm{mm})$. Déc. = décapage ; $N=$ nombre de vestiges récupérés (fouille et tamisage).

Table 3 - Isturitz, Saint-Martin room. Proportions of lithic remains by dimensional class recovered from the two samplecolumns. Dimensional classes are expressed in mesh width. Déc. = exposed surface; $N=$ number of gathered remains (excavation and sieving).

indique que ces tris représentent très probablement des objets déposés en zone distale de ruissellement concentré ou à la suite de brefs épisodes de fonctionnement ;

- les tris de l'unité III, en particulier ceux observés en $V_{1} 29 \mathrm{~b}$, s'accordent avec des redistributions soit par un ruissellement peu compétent en dehors des rigoles, soit par ruissellement concentré. En $V_{1} 31 b$, des épisodes de pavages résiduels peuvent également être à l'origine d'une grande partie des tris observés. Dans ce carré, deux décapages du sommet de l'unité présentent un tri qui signe une redistribution. Le premier correspond au faciès de pseudo-sables brun jaune (décapage 16). Le second se place dans une zone de transition où le diamicton est à support matriciel, intercalé dans le faciès de pseudo-sables (décapage 19).
Cette information est complétée par la prise en compte de l'évolution verticale de l'abondance en matériel lithique (fig. 12). Celle-ci fait apparaître deux informations. Premièrement, on observe un contraste de richesse entre les unités II et III, ainsi qu'une diminution en matériel archéologique au sommet de l'unité III qui se poursuit dans l'unité II. Deuxièmement, une forte variabilité de l'unité IIII y est mise en évidence : la nappe de vestiges est constituée d'horizons successivement riches et pauvres en vestiges. En outre, l'épaisseur des passées triées de l'unité III est pluricentimétrique à décimétrique, c'est-à-dire comparables à l'épaisseur des lentilles litées observées dans cette unité. Enfin, aucun tri n'a été reconnu pour les passées les plus riches. 

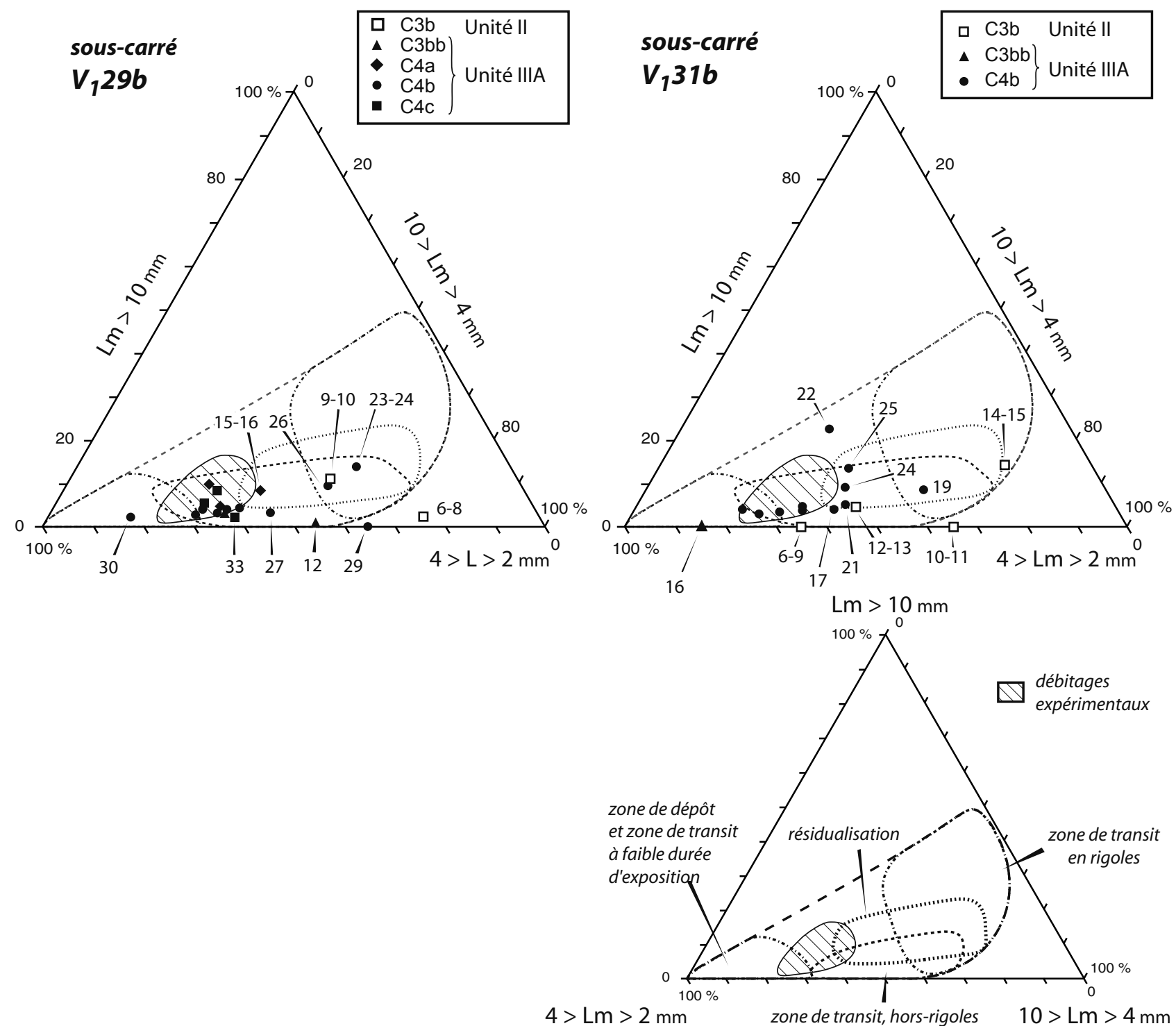

Figure 11 - Triangle des classes dimensionnelles. Les enveloppes portées sont celles du référentiel expérimental (en bas à droite). Les numéros des mesures sont ceux des décapages. Pour préserver la lisibilité de la figure, les décapages successifs qui présentent des proportions identiques de classes de taille sont regroupés, ainsi que les décapages à effectif jugé trop faible $(N<20)$.

Figure 11 - Triangular texture diagram. Areas drawn on the diagram have been established from an experimental model (lower right). The measurement numbers correspond to those of the exposed surfaces. For clarity purposes, the successive exposed surfaces, which comprise the same dimensional classes, are put together as well as the exposed surfaces whose strength is thought to be too weak.

\section{2 - Interprétation}

Dans I'unité II, la faible proportion de sédiments anthropiques, l'évidence de tris granulométriques et la pauvreté en matériel lithique indiquent un apport en vestiges redistribués par ruissellement. La raréfaction en vestiges et en sédiment archéologique au sommet de l'unité III indique que la sédimentation naturelle s'est poursuivie après l'abandon de la salle par les préhistoriques. Les quelques pièces redistribuées recueillies dans une masse de sédiment naturel ne semblent alors représenter que des objets initialement contenus dans l'unité III qui pouvaient affleurer latéralement ou être érodés par les incisions du ruissellement.
Au sein de l'unité III, une distinction doit être faite entre les zones à forte éboulisation (unité Illa secteur sud) et les zones où le ruissellement est l'agent exclusif de sédimentation (unité Illa secteur nord et unité IIIb). Dans le diamicton, la fabrique est celle d'un dépôt gravitaire. Les gros vestiges, à l'image de ceux dont l'attitude est mesurée (longueur $>2 \mathrm{~cm}$ ), n'ont donc pas été affectés par le ruissellement. Les tris granulométriques observés s'accordent avec cette interprétation dans la mesure où ils signent des épisodes de formation de pavages résiduels. De tels pavages ont par ailleurs été mis au jour au sommet du niveau IIlc, qui présente un lithofaciès comparable de diamicton à support clastique. Les ensembles de vestiges contenus dans ces lithofaciès sont biaisés par exportation des 


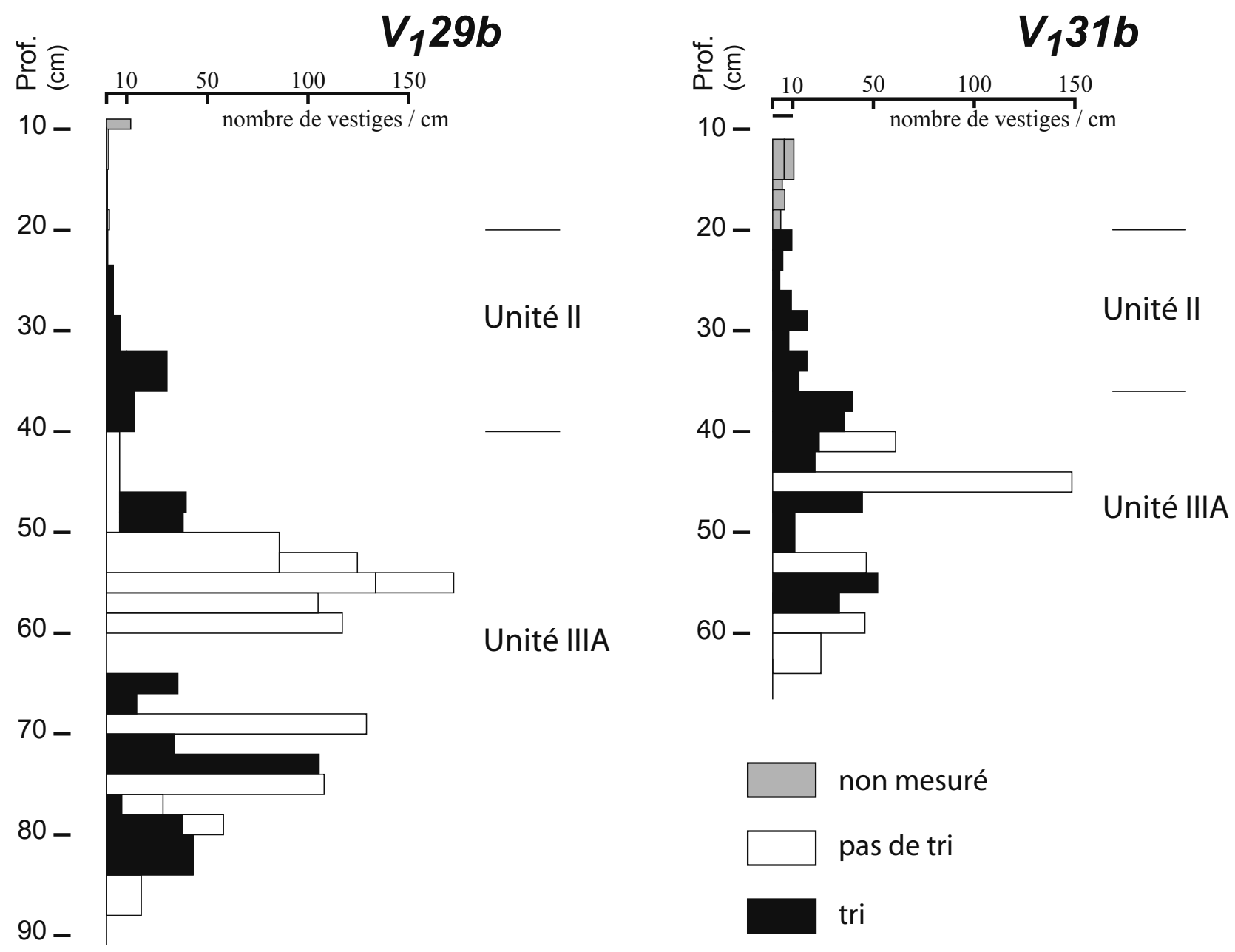

Figure 12 - Isturitz, salle Saint-Martin. Evolution verticale du tri granulométrique et de l'abondance en silex taillé au sein des unités II et IIla.

Figure 12 - IIsturitz, Saint-Martin room. Vertical evolution of sorting and archaeological richness of unit Illa deposits.

objets les plus petits ; mais la position stratigraphique des objets qui y sont retrouvés ne semble pas devoir être remise en question.

En dehors des zones d'éboulis, les épisodes de ruissellements compétents mis en évidence par l'étude des sédiments ont contribué significativement à la formation des nappes de vestiges. Cela est montré par les passées à tri prononcé et à fabrique diagnostique. Ces horizons de matériels redistribués s'intercalent entre des passées sans tri ni orientation préférentielle. La richesse en matériel archéologique de ces dernières peut indiquer qu'il s'agit de dépôts non redistribués par les agents naturels ou refléter le caractère parfois non diagnostique des critères utilisés.

\section{3 - Implications sur la signification des séries recueillies à la fouille}

Plusieurs implications se dégagent de l'étude réalisée.
Premièrement, la solifluxion n'a pas eu d'impact significatif sur la constitution des nappes de vestiges. En effet, toutes les modifications reconnues (organisations remarquables, orientation des vestiges ou tris dimensionnels) sont imputables à l'action du ruissellement.

Deuxièmement, une partie importante des dépôts en cours de fouille dans la salle Saint-Martin livre des passées de matériel en position secondaire. Cela n'est cependant pas le cas des lithofaciès caillouteux de l'unité Illa, présents dans la partie sud de la fouille principale (rangées 31 et 32) et au-delà vers le sud (rangées 33 à 39). Là, les modifications reconnues sont mineures. Elles ne sont pas de nature à invalider la relation d'équivalence entre la succession des nappes de vestiges et des occupations préhistoriques. Sur la base de la similitude des dynamiques sédimentaires à l'origine du dépôt, cette interprétation peut également être étendue au niveau Illc. Les données 
nouvelles qu'apportera la poursuite de la fouille seront utiles pour valider ce dernier point.

Les redistributions identifiées dans les autres faciès où le ruissellement domine ont nécessairement des conséquences sur l'enregistrement archéostratigraphique et donc, a fortiori, sur les ensembles recueillis à la fouille. Ces distorsions peuvent être appréhendées sur la base du modèle établi par Lenoble (2005). Selon celui-ci, les redistributions qui accompagnent l'enfouissement des vestiges peuvent avoir deux conséquences. La première est celle d'un enrichissement des couches archéologiques par apport de matériel déplacé contemporain ou antérieur aux occupations préhistoriques. La seconde consiste dans la constitution de pseudo-niveaux archéologiques. Dans les deux cas, il en résulte une diffusion du matériel de chaque occupation dans les niveaux sus-jacents. Les industries recueillies au sein de ces lithofaciès peuvent donc donner l'illusion de la persistance d'un trait culturel, alors qu'il ne s'agit que d'éléments redistribués.

Un tel constat conduit nécessairement à s'interroger sur l'homogénéité des nappes de vestiges incluses dans les pseudo-sables lités de l'unité III.

Toutefois, le travail réalisé n'est pas de nature à quantifier les mélanges entre industries. Sont-ils négligeables, significatifs ou majoritaires ? Des études complémentaires sont nécessaires pour répondre à cette question. Ce peut être, par exemple, la recherche de raccords inter-couches, la distribution des matières premières rares, etc.

\section{Conclusions}

La cavité d'Isturitz correspond à l'étage supérieur d'un réseau karstique comportant trois niveaux. Plusieurs phases évolutives y ont été identifiées : 1) creusement de la grotte en contexte phréatique, 2) façonnement par l'Arberoue (dépôts d'alluvions torrentielles) et édification d'importants massifs stalagmitiques, 3) écroulement de la partie sud-ouest de la cavité et, peut-être, retour à des conditions phréatiques, 4) mise en place en contexte vadose des dépôts détritiques et phosphatés qui contiennent les industries paléolithiques, 5) formation de spéléothèmes qui, dans la plus grande partie de la cavité, scellent les dépôts précédents. La situation chronologique des trois premières phases n'a pu être précisée ; elles se placent probablement entre le début du Quaternaire et la fin du Pléistocène moyen. La quatrième date du Pléistocène supérieur et la dernière de l'Holocène.

Les coupes actuellement observables dans la salle SaintMartin concernent ces deux derniers épisodes évolutifs. Elles permettent de constater que la sédimentation témoigne d'abord d'écoulements de haute énergie, lors des occupations moustériennes (unité IV), qu'elle est ensuite dominée par le ruissellement lors des occupations protoaurignaciennes et aurignaciennes (unité III) et, enfin, par l'éboulisation associée au ruissellement (unité II). Un gélisol superficiel accompagne l'édification des dépôts ruisselés tandis qu'un gélisol profond, peut-être un pergélisol, se développe postérieurement à la formation des dépôts détritiques et avant celle du plancher stalagmitique supérieur (unité I).

D'après les études spécifiques réalisées, les manifestations du gel ne semblent avoir eu que peu d'incidence sur les nappes de vestiges, au moins dans la zone fouillée. Ce n'est, en revanche, pas le cas du ruissellement. En effet, les objets archéologiques présents dans l'unité II sont probablement liés au fonctionnement de ce mécanisme ; ils correspondent sans doute à des remaniements en provenance de niveaux archéologiques situés dans l'unité III. Dans l'unité III, l'impact du ruissellement est variable à la fois latéralement et verticalement. Des secteurs fortement affectés côtoient des zones peu, ou même, très peu modifiées. Dans ce dernier cas, on constate un simple appauvrissement en pièces de petit calibre ; ce processus n'est alors pas de nature à modifier significativement l'enregistrement archéostratigraphique.

\section{Remerciements}

Nous remercions Christian Normand et Alain Turq, responsables de l'opération de fouille, qui ont rendu possible la réalisation de cette étude en facilitant l'accès au terrain et en permettant de bénéficier de l'infrastructure et des moyens financiers de l'opération. Nous remercions également Mathieu Rué et Dominique Todisco pour leur relecture rigoureuse.

\section{Références bibliographiques}

BACSHELET E. 1981 - Circular statistics in biology. Londres : Academic Press, $374 \mathrm{p}$.

BENN D.I. 1994 - Fabric shape and the interpretation of sedimentary fabric data. Journal of Sedimentary Research, A64, 4, p. 910-915.

BERRISFORD M.S. 1991 - Evidence for enhanced mechanical weathering associated with seasonally latelying snow patches, Jotunheimen, Norway. Permafrost andcomparative Periglacial Processes, 2, p. 331-340.

BERTRAN P. 1999 - Dynamique des dépôts de la grotte Bourgeois-Delaunay (La Chaise-de-Vouthon, Charente) : apport de la micromorphologie. Paleo, 11, p. 9-18.

BERTRAN P., CLAUD E., DETRAIN L., LENOBLE A., MASSON B. et VALLIN L. 2006 - Composition granulométrique des assemblages lithiques, application à l'étude taphonomiques des sites paléolithiques. Paleo, 18, p. 7-35.

BERTRAN P. et LENOBLE A. 2002 - Fabriques des niveaux archéologiques : méthode et premier bilan des apports à l'étude taphonomique des sites paléolithiques. Paleo, 14, p. 13-28.

BOISSONNAS J., DESTOMBES J.P., HEDDEBAUT CL., LE POCHAT G., LORSIGNOL S., ROGER Ph., TERNET Y. et THIBAULT Cl. 1974 - Carte géologique à 1/50 000 d'Iholdy. Orléans : B.R.G.M., service géologique national. 
BRETZ J.H. 1942 - Vadose and phreatic features of limestone caverns. The Journal of Geology, 50, 6, p. $675-811$.

BULLOCK P., FÉDOROFF N., JONGERIUS A., STOOPS G. et TURSINA T. 1985 - Handbook for soil thin section description. Worlverhampton : Waine Research Publications, $152 \mathrm{p}$.

CAMPY M. et CHALINE J. 1993 - Missing records and depositional breaks in French late Pleistocene cave sediments. Quaternary Research, 40, p. 318-331.

GUILLORÉ P. 1980 - Méthode de fabrication mécanique et en série des lames minces. Paris : Institut National Agronomique (Département des Sols), $22 \mathrm{p}$.

HORWITZ L. K. et GOLDBERG P. 1989 - A study of Pleistocene and Holocene hyena coprolites. Journal of Archaeological Science, 16, p. 71-94.

LARRIBAU J.-D. ET PRUDHOMME S. 1983 - La grotte ornée d'Erberua (Pyrénées-Atlantiques), note préliminaire. Bulletin de la société préhistorique française, 80, 9, p. $280-284$.

LAUTRIDOU J.-P. 1976 - Les expériences de cryoclastie. Bulletin du Centre de Géomorphologie du CNRS de Caen, 21, p. 21-28.

LENOBLE A. 2005 - Ruissellement et formation des sites préhistoriques : référentiel actualiste et exemples d'application au fossile. Oxford: British Archaeological Report International Series, $n^{\circ} 1363,212 \mathrm{p}$.

LENOBLE A., BERTRAN P., MERCIER N. et SITZIA L. 2012 - Le site du Lac bleu et la question de l'extension du pergélisol en France au Pléistocène supérieur. In : $P$. Bertran et A. Lenoble (Eds.), Quaternaire continental d'aquitaine : un point sur les travaux récents. Livret-guide de l'excursion de l'AFEQ. Talence : imprimerie de l'université de Bordeaux 1, p. 109-121.

LUNDBERG J. ET MCFARLANE D.A. 2009 - Bats and bell holes: The microclimatic impact of bat roosting, using a case study from Runaway Bay Caves, Jamaica. Geomorphology, 106, p. 78-85.

MARK D.A. 1973 - Analysis of axial orientation data, including till fabrics. Geological Society of America Bulletin, 84, p. 1969-1974.

MARTINI J. E. 2000 - La grotte et le karst de Cango, Afrique du Sud. Karstologia, 36, 2, p. 43-54.

MATSUOKA N. 1991 - A model of the rate of frost shattering: Application to field data from Japan, Svalbard and Antarctica. Permafrost and Periglacial Processes, 2, p. 271-281.

McEACHRAN D.B. 1990 - Stereo, the stereographic projection programme. Apple Macintosh Computer Software, version 1.3.

NORMAND C. 2002 - Grotte d'Isturitz, salle de SaintMartin (Commune de Saint-Martin d'Arberoue). Rapport final de fouilles programmées triannuelles. Bordeaux : Service Régional de l'Archéologie d'Aquitaine, 115 p.

NORMAND C., BEAUNE S. de, COSTAMAGNO S., DIOT M.-F., HENRY-GAMBIER D., GOUTAS N., LAROULANDIE V., LENOBLE A., O'FARRELL M., RENDU W., RIOS GARAIZE J., SCHWAB C., TARRINO VINAGRE A., TEXIER J.-P. et WHITE R. 2007 - Nouvelles données sur la séquence aurignacienne de la grotte d'Isturitz (commune d'Isturitz et de Saint-Martind'Arberoue ; Pyrénées-Atlantiques). In : J. Evin (dir.), Un siècle de construction du discours scientifique en Préhistoire. Actes du $\left.\mathrm{XXV}\right|^{e}$ Congrès Préhistorique de France. Paris : Société Préhistorique française, vol. III, p. 277-293.

PASSEMARD E. 1944 - La grotte d'Isturitz. Paris : Presses universitaires, $95 \mathrm{p}$.

SAINT-PÉRIER R. (de) 1936 - La grotte d'Isturitz II : le Magdalénien de la Grande Salle. Archives de l'Institut de Paléontologie Humaine, 17. Paris : Masson, 139 p.

SAINT-PÉRIER R. et S. (de) 1952 - La grotte d'Isturitz III : les Solutréens, les Aurignaciens et les Moustériens. Archives de l'Institut de Paléontologie Humaine, 25. Paris : Masson, $310 \mathrm{p}$.

SZMIDT C., NORMAND C., BURR G.S., HODGINS G.W.L. et LAMOTTA S. 2010 - AMS 14C dating the Protoaurignacian/Early Aurignacian of Isturitz, France. Implications for Neanderthal-modern human interaction and the timing of technical and cultural innovations in Europe. Journal of Archaeological Science, 37, 4, p. 758-768.

TEXIER J.-P. 1996 - Présence d'un réseau de grands polygones au sud de l'estuaire de la Gironde (France) : interprétation et implications paléoclimatiques. Géographie Physique et Quaternaire, 50, 1, p. 103-108.

TEXIER J.-P. et BERTRAN P. 1993 - Données nouvelles sur la présence d'un pergélisol en Aquitaine au cours des dernières glaciations. Permafrost and Periglacial Processes, 4, 3, p. 183-198.

VAN VLIET-LANOË B. 1988 - Le rôle de la glace de ségrégation dans les formations superficielles de l'Europe de l'Ouest. Processus et héritages. Thèse d'Etat, Université de Paris I - Sorbonne, 854 p.

WATSON G.S. 1966 - The statistics of orientation data. Journal of Geology, 74, p. 786-797.

WHITE W.B., 1988 - Geomorphology and Hydrology of Karst Terrains. Oxford : Oxford University Press, 464 p.

WOODCOCK N.H. 1977 - Specification of fabric shapes using an eigenvalue method. Geological Society of America Bulletin, 88, p. 1231-1236. 